\title{
Hall and Ion-Slip Currents Effects on Unsteady MHD Fluid Flow over an Inclined Plate with Inclined Magnetic Field and Variable Temperature
}

Mohammad Rafiqul Islam ( $\nabla$ mribsmrstu@yahoo.com )

Bangabandhu Sheikh Mujibur Rahman Science and Technology University

Sonia Nasrin

Jagannath University

\section{Research Article}

Keywords: MHD fluid, Heat transfer, Mass transfer, Inclined angle, Hall and Ion-slip current

Posted Date: May 5th, 2021

DOl: https://doi.org/10.21203/rs.3.rs-489560/v1

License: (c) (1) This work is licensed under a Creative Commons Attribution 4.0 International License.

Read Full License 


\title{
Hall and Ion-Slip Currents Effects on Unsteady MHD Fluid Flow over an Inclined Plate with Inclined Magnetic Field and Variable Temperature
}

\author{
M. R. Islam ${ }^{1 *}$ and S. Nasrin ${ }^{2}$ \\ ${ }^{1}$ Department of Mathematics, Bangabandhu Sheikh Mujibur Rahman Science and \\ Technology University, Gopalganj-8100, Bangladesh \\ ${ }^{2}$ Department of Mathematics, Jagannath University, Dhaka-1100, Bangladesh \\ *Corresponding author email: mribsmrstu@yahoo.com
}

\begin{abstract}
A numerical study is performed to investigate the effect of Hall and Ion-slip current on unsteady MHD viscous electrically conducting fluid over an electrically non-conducting inclined plate, which is along the plate under the strong inclined magnetic field. The magnetic Reynolds number of flows is taken very small so that the induced magnetic field equation is ignored in our analysis. The governing equations are derived from the NavierStokes equation, Energy equation and Concentration equation and boundary layer approximation has been employed. The obtained non-linear, nondimensional coupled governing equations are solved by using numerical methods of explicit finite difference for velocity, temperature and concentration. The flows are affected by the inclined angle. The effect of necessary important relevant parameters on the velocity, temperature and concentration distributions has been discussed in detail and also the shear stress, Nusselt number and Sherwood number are explained and compare it with its natural behavior, as well as graphically.
\end{abstract}

Keywords: MHD fluid, Heat transfer, Mass transfer, Inclined angle, Hall and Ion-slip current.

\section{Introduction}

The influence of the magnetic field of MHD fluid flows with hall and ion slip current has a great importance of experimental and theoretical applications in magnetic material processing, astrophysics, nuclear engineering, geophysical and industrial fields. Abel and Veena (1998) discussed the visco-elastic fluid flow in a saturated porous medium with internal heat generation and frictional heating over a stretching surface. A free- streamline theory is carried out by Abernathy (1962) for a flat plate of inclination towards the infinite flow field at an angle of attack. Measurement of the position of the free-rotation layers, the frequency of the rotation street and back-pressure plate of a sharp-edged plate have been reported to be effective both in attacking angle and lateral contraction of the flow. Ajay (2003) explained the combined effects of hall current, viscous dissipation, Joule heating and thermal diffusion on the magneto-hydrodynamic free convection flow of an electrically conducting fluid with mass transfer past an infinite vertical porous plate. Angirasa and Peterson (1997) presented a numerical study on heat transfer in natural transmission from an isothermal vertical surface which is a stable layered to a fluid-saturated thermally stratified porous medium. Basant and Malgwi (2019) have analyzed hall and ion-slip current effect on free convection of an incompressible viscous fluid flow in a vertical microchannel with an induced magnetic field. Beg et al. (2010) have developed a numerical solution for MHD viscous, incompressible flow in a rotating porous channel comprising two infinite parallel plates with hall currents and inclined magnetic field. Bhpendra et al. (2007) presented the hall current effect on the unsteady viscous incompressible mixed convective fluid flow past a vertical infinite porous plate with the heat source (or sink). Two immiscible convective flow and heat transfer with porous past an inclined channel with pressure gradient is discussed by Daniel and Daniel (2013). Eraslan (1969) expressed the fully developed distributions of temperature for the MHD channels with Hall Effect. They have found the presence of porous structure, material constants and different liquids can efficiently control fluid flow. Gupta (1997) studied the heat and mass transfer of an electromagnetic fluid flow on a stretching sheet with suction as well as with blowing. Javeri (1975) investigated the effect of combined hall, ion slip, Joule heating and viscous dissipation on the laminar MHD channel heat transfer. Jyotsna et al. (2017) have analyzed the MHD heat and mass transfer free convection flow past an exponentially accelerated inclined plate embedded in a saturated porous medium with radiation and mass transfer effect. Kumar and Singh (1999) analyzed the natural convection of heat transfer in a poachy porous medium from a vertically isothermal surface under the influence of thermal stratification. Navnet et al. (2016) examined the inclined magnetic field effect on unsteady viscous, incompressible fluid flow past on a vertical moving plate with variable temperature. Opanuga et al. (2018) have investigated the rate of entropy generation on a couple of stress fluids in the presence of hall and ion-slip current with velocity slip and jump of temperature. The influence of hall current of the generalized Couette flow of a viscous, conducting fluid under an inclined magnetic field is analyzed by Prasada et al.(1986). Rajput and Gupt (2016) have considered the unsteady free convection MHD fluid flow along an exponentially accelerated plate past in a porous medium with inclined magnetic field, variable temperature and constant mass diffusion. Ram (1995) considered the hall and ion-slip current effects of free convective heat-generating rarefied gas in a rotating frame with a strong magnetic field imposed perpendicular to the plate. Rishab et al. (2017) have presented the flow of visco-elastic electrically conducting fluid through two parallel plates filled with a porous medium placed in an inclined magnetic field with heat and mass transfer. Sato has discussed the electrical conductivity of an ionized gas with the Hall Effect, where the conductivity is presented by a tensor in a similar form for both fully and partially ionized gases. Seth et al. $(1961,2009,2010,2016)$ have studied the Hall Effect in the Viscous Flow of Ionized Gas Between two Parallel Plates under Transverse Magnetic Field; MHD Couette Flow in the presence of an inclined magnetic field with rotation; Hartmann's flow of a viscous incompressible electrically conducting fluid in the presence of the inclined magnetic field with hall effects and with rotation and also studied on the MHD free convection flow of a viscous, incompressible, electrically 
conducting fluid past an impulsively started vertical plate through a saturated porous medium with ramped heat flux in the presence of the inclined magnetic field. Suman and Nazibuddin (2018) presented the unsteady MHD flow with mass transfer along an infinite inclined plate where the velocity is strung in a poachy porous medium with a variant temperature.

Recently Krishna et al. (2020) have modified the work of Jyotsna et al. and investigated the hall and ion slip current effects on unsteady magneto-hydrodynamic free convective flow with rotation through an exponentially accelerated inclined plate entrenched in a saturated porous medium with the effect of inclined angle also with the change of reference frame. They have used Laplace transform to solve these problems analytically. In this paper, our main aim is to modify the work of Jyotsna et al. This study presented the result of numerical modeling of the unsteady MHD fluid flow over an inclined plate and inclined induced magnetic field without change of reference frame with time variation measurements by the effect of Dufour, hall and ion-slip currents.

\section{Mathematical Model}

Let an electrically non-conducting plate be fixed at $y=0$ in the fluid which makes an angle $\alpha$ with $x$ - axis vertically upward and $y$-axis is normal to the $x z$-plane. Consider an unsteady MHD incompressible viscous electrically conducting fluid flow in the direction of parallel to the plate. Initially, we assumed that fluid velocity, temperature and concentration of the fluid are respectively $u=0, \quad w=0, \quad T=T_{\infty}$ and $C=C_{\infty}$. At the time $t>0$, the plate oscillates in its plane with a velocity $U_{0} \cos \omega t$ along the plate and the temperature of the plate and the concentration are raised from $T_{\infty}+\left(T_{w}-T_{\infty}\right) \frac{U_{0}^{2} t}{v}$ to $T_{\infty}$ and $C_{\infty}+\left(C_{w}-C_{\infty}\right) \frac{U_{0}^{2} t}{v}$ to $C_{\infty}$ with time $t$. Due to the effect of hall and Ion-slip current, the generalized Ohm's law may state as follows: $\mathbf{J}+\frac{\beta_{e}}{B_{0}}(\mathbf{J} \wedge \mathbf{B})=\sigma(\mathbf{q} \wedge \mathbf{B})+\frac{\omega_{e} \tau_{e} \beta_{i}}{B_{0}^{2}}(\mathbf{J} \wedge \mathbf{B}) \wedge \mathbf{B}$

Where $\omega_{e} \tau_{e}=\beta_{e}$ may treat as Hall parameter. We assumed that the magnetic field $\mathbf{B}$ is imposed for the electrically non-conducting plate at a right angle to the plate. The magnetic Reynolds number of flows is taken very small, so that induced magnetic field equation is not considerable in our analysis. And therefore the induced magnetic field vector becomes

$\mathbf{B}=\left(-B_{0} \sin \alpha, B_{0} \cos \alpha, 0\right)$

Physical model of the system is shown in the following Fig.1.

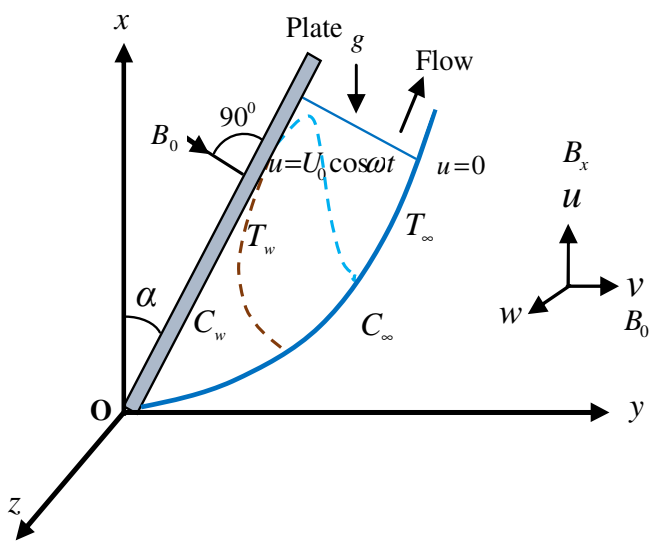

Fig.1 Physical configuration and coordinate system

It is assumed that the plate is of semi-infinite extended length, then all the physical variables in the problem are functions of $x$, $y$ and $t$. Therefore the problem is two-dimensional. According to the above-mentioned assumptions along with the Boussinesq and boundary layer approximations, the governing equations of fluid and generalized Ohm's law with Hall current and ion-slip influence are given as follows:

$$
\begin{aligned}
& \frac{\partial u}{\partial x}+\frac{\partial v}{\partial y}=0 \\
& \frac{\partial u}{\partial t}+u \frac{\partial u}{\partial x}+v \frac{\partial u}{\partial y}=g \beta \cos \alpha\left(T-T_{\infty}\right)+g \beta^{*} \cos \alpha\left(C-C_{\infty}\right)+v \frac{\partial^{2} u}{\partial y^{2}}-\frac{\sigma B_{0}^{2}}{\rho}\left(\Gamma_{1} u+\Gamma_{2} w\right) \\
& \frac{\partial w}{\partial t}+u \frac{\partial w}{\partial x}+v \frac{\partial w}{\partial y}=v \frac{\partial^{2} w}{\partial y^{2}}+\frac{\sigma B_{0}^{2}}{\rho}\left(\Gamma_{2} u-\Gamma_{3} w\right) \\
& \frac{\partial T}{\partial t}+u \frac{\partial T}{\partial x}+v \frac{\partial T}{\partial y}=\frac{k}{\rho c_{p}} \frac{\partial^{2} T}{\partial y^{2}}+\frac{D_{m} k_{T}}{c_{s} c_{p}} \frac{\partial^{2} C}{\partial y^{2}} \\
& \frac{\partial C}{\partial t}+u \frac{\partial C}{\partial x}+v \frac{\partial C}{\partial y}=D_{m} \frac{\partial^{2} C}{\partial y^{2}}
\end{aligned}
$$


with corresponding boundary conditions:

$$
\begin{gathered}
t \leq 0: \quad u=0, \quad w=0, \quad T=T_{\infty}, \quad C=C_{\infty} \\
t>0:\left\{\begin{array}{c}
u=U_{0} \cos \omega t, w=0, \quad T=T_{\infty}+\left(T_{w}-T_{\infty}\right) \frac{U_{0}^{2} t}{v} \\
u=0, \quad w=0, \quad T \rightarrow T_{\infty}, \quad C \rightarrow C_{\infty} \text { as } y \rightarrow \infty \\
\text { where, } \Gamma_{1}=\frac{\left(1+\beta_{e} \beta_{i} \cos \alpha\right) \cos ^{2} \alpha}{\left(1+\beta_{e} \beta_{i}\right)\left(1+\beta_{e} \beta_{i} \cos ^{2} \alpha\right)+\beta_{e}^{2} \cos ^{2} \alpha}, \\
\Gamma_{2}=\frac{\beta_{e} \cos ^{3} \alpha}{\left(1+\beta_{e} \beta_{i}\right)\left(1+\beta_{e} \beta_{i} \cos ^{2} \alpha\right)+\beta_{e}^{2} \cos ^{2} \alpha} \\
\text { and } \Gamma_{3}=\frac{\left(1+\beta_{e} \beta_{i}\right) \cos ^{2} \alpha}{\left(1+\beta_{e} \beta_{i}\right)\left(1+\beta_{e} \beta_{i} \cos ^{2} \alpha\right)+\beta_{e}^{2} \cos ^{2} \alpha}
\end{array}\right.
\end{gathered}
$$

\section{Similarity Analysis}

The following non-dimensional variables are introduced to make the dimensionless form of the above equations.

$$
x^{*}=\frac{U_{0}}{v} x, y^{*}=\frac{U_{0}}{v} y, u^{*}=\frac{u}{U_{0}}, v^{*}=\frac{v}{U_{0}}, w^{*}=\frac{w}{U_{0}}, \omega^{*}=\frac{v}{U_{0}^{2}} \omega, t^{*}=\frac{U_{0}^{2}}{v} t, \theta=\frac{T-T_{\infty}}{T_{w}-T_{\infty}}, \phi=\frac{C-C_{\infty}}{C_{w}-C_{\infty}}
$$

Removing the asterisk sign, the non-dimensional form of the Continuity equation, Momentum equations, Energy equation and Concentration equation as follows:

$$
\begin{aligned}
& \frac{\partial u}{\partial x}+\frac{\partial v}{\partial y}=0 \\
& \frac{\partial u}{\partial t}+u \frac{\partial u}{\partial x}+v \frac{\partial u}{\partial y}=G_{r} \theta \cos \alpha+G_{m} \phi \cos \alpha+\frac{\partial^{2} u}{\partial y^{2}}-H_{a}\left(\Gamma_{1} u+\Gamma_{2} w\right) \\
& \frac{\partial w}{\partial t}+u \frac{\partial w}{\partial x}+v \frac{\partial w}{\partial y}=\frac{\partial^{2} w}{\partial y^{2}}+H_{a}\left(\Gamma_{2} u-\Gamma_{3} w\right) \\
& \frac{\partial \theta}{\partial t}+u \frac{\partial \theta}{\partial x}+v \frac{\partial \theta}{\partial y}=\frac{1}{P_{r}} \frac{\partial^{2} \theta}{\partial y^{2}}+D_{f} \frac{\partial^{2} \phi}{\partial y^{2}} \\
& \frac{\partial \phi}{\partial t}+u \frac{\partial \phi}{\partial x}+v \frac{\partial \phi}{\partial y}=\frac{1}{S_{c}} \frac{\partial^{2} \phi}{\partial y^{2}}
\end{aligned}
$$

With the corresponding boundary conditions:

$$
\begin{aligned}
& t \leq 0: \quad u=0, w=0, \quad \theta=0, \phi=0 \\
& t>0:\left\{\begin{array}{l}
u=\cos \omega t, w=0, \quad \theta=t, \phi=t \text { at } y=0 \\
u=0, w=0, \quad \theta \rightarrow 0, \phi \rightarrow 0 \text { as } y \rightarrow \infty
\end{array}\right.
\end{aligned}
$$

where, $G_{r}=\frac{v g \beta}{U_{0}^{3}}\left(T_{w}-T_{\infty}\right)=$ Grashof number; $G_{m}=\frac{v g \beta^{*}}{U_{0}^{3}}\left(C_{w}-C_{\infty}\right)=$ Solutal Grashof number; $H_{a}=\frac{v \sigma B_{0}^{2}}{\rho U_{0}^{2}}=$ Hartmann number; $P_{r}=\frac{\rho c_{p} v}{k}=$ Prandtl number; $D_{f}=\frac{D_{m} k_{T}}{v c_{s} c_{p}} \frac{\left(C_{w}-C_{\infty}\right)}{\left(T_{w}-T_{\infty}\right)}=$ Dufour number; $S_{c}=\frac{v}{D_{m}}=$ Schmidt number.

\section{Method of Solutions}

The dimensionless, nonlinear, coupled partial differential equations (6)-(10) together with associated boundary conditions are solved by using the explicit finite difference method. The maximum length of the plate is taken $x_{\max }(=40)$ and the maximum length of the boundary layer is $y_{\max }(=25)$ corresponding to $y \rightarrow \infty$. This means $x$ varies from 0 to 40 and $y$ varies from 0 to 25 . The finite difference schemes for some variables with respect to $t, x$ and $y$ as follows:

$$
\frac{\partial u}{\partial t}=\frac{U_{i, j}^{k+1}-U_{i, j}^{k}}{\Delta t} ; \quad \frac{\partial u}{\partial x}=\frac{U_{i, j}^{k}-U_{i-1, j}^{k}}{\Delta x} ; \quad \frac{\partial u}{\partial y}=\frac{U_{i, j}^{k}-U_{i, j-1}^{k}}{\Delta y} ; \quad \frac{\partial^{2} u}{\partial y^{2}}=\frac{U_{i, j+1}^{k}-2 U_{i, j}^{k}+U_{i, j-1}^{k}}{(\Delta y)^{2}}
$$

Here, the subscript ' $i$ ' refers to $x$; ' $j$ ' to $y$ and superscript ' $k$ ' refers to time $t$. Similarly, we have used finite difference schemes for the other variables. We have used Matlab code to get the solution of finite difference schemes and its graphical presentation.

\section{Shear stresses, Nusselt number and Sherwood number:}

The effects of various parameters on local and average shear stress have been investigated from the velocity profile. The velocity gradient at the plate is defined as the shear stress; the non-dimensional form of the local shear stress and average shear stress in $x$ - 
direction are given by the relations $\tau_{L}=\left.\mu \frac{\partial u}{\partial y}\right|_{y=0}$ and $\tau_{A}=\left.\frac{1}{L} \int_{0}^{L} \mu \frac{\partial u}{\partial y}\right|_{y=0} d x$ respectively. From the temperature profile, the effects of various parameters on local and average Nusselt number have been calculated. The rate of heat transfer at the plate is defined as the Nusselt number; the local Nusselt number and the average Nusselt number are given by $N u_{L}=-\left.\mu \frac{\partial \theta}{\partial y}\right|_{y=0}$ and $N u_{A}=-\left.\frac{1}{L} \int_{0}^{L} \mu \frac{\partial \theta}{\partial y}\right|_{y=0} d x$ respectively. Similarly, we have analyzed the effects of various parameters on local and average Sherwood number from the concentration field. The rate of mass transfer at the plate is defined as the Sherwood number; the local Sherwood number and the average Sherwood number are defined by $S h_{L}=-\left.\mu \frac{\partial \phi}{\partial y}\right|_{y=0}$ and $S h_{A}=-\left.\frac{1}{L} \int_{0}^{L} \mu \frac{\partial \phi}{\partial y}\right|_{y=0} d x$ respectively.

\section{Results and Discussions}

The results carried for the influence of the relevant non-dimensional parameters namely Hartmann number $\left(H_{a}\right)$, thermal Grashof number $\left(G_{r}\right)$, solutal Grashof number $\left(G_{m}\right)$, Hall parameter $\left(\beta_{e}\right)$, ion-slip parameter $\left(\beta_{i}\right)$, Prandtl number $\left(P_{r}\right)$, Dufour number $\left(D_{f}\right)$ and Schmidt number $\left(S_{c}\right)$ on various distributions such as on the velocity, temperature, concentration, local and average shear stress, local and average Nusselt number, local and average Sherwood number. The effects of those parameters on some important profiles are shown in Figs.4-36.

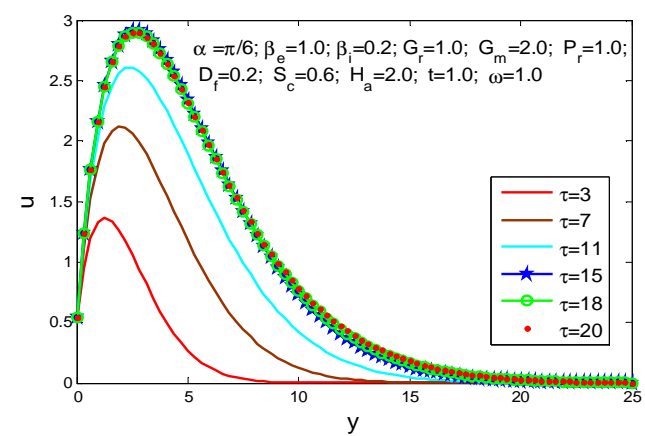

Fig.2: Time sensitivity on $u$ for different values of maximum time ' $\tau$ ',

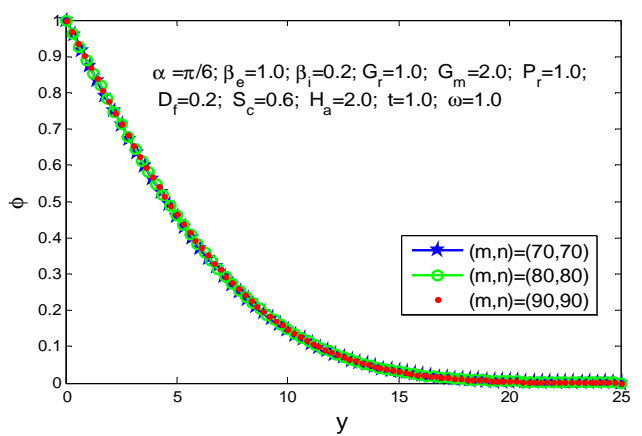

Fig.3: Mesh sensitivity on $\varphi$ for different values of mesh spaces $(m, n)$

\section{Time and Mesh Sensitivity Test}

For the time sensitivity, the computations have been carried out for different maximum time $t=3,7,11,15,18$ and 20 with time increment $\Delta t=0.01$ on the velocity profile which is shown in Fig.2. It has seen from the figure that the velocities are very minimal change after $t=15$, therefore the steady-state solutions are considered for the time $t=15$ with time increment $\Delta t=0.01$. Also for mesh sensitivity, the computations have been carried out for different mesh spaces such as $(m, n)=(70,70) ;(m, n)=(80,80)$ and $(m, n)=(90,90)$ within the intervals $0 \leq x \leq 40 \quad \& 0 \leq y \leq 25$ on the concentration, which is shown in Fig. 3 . It has observed from figure that there is also a negligible change with different meshes. From those pairs of mesh points, we have chosen $(m, n)=(80,80)$ for our computations. The same situation for the other distributions for steady-state time and mesh.

\section{Primary Velocity Distributions}

At first, it is mentioned from our physical configuration of the model that "the plate is inclined means induced magnetic field is also inclined because induced magnetic field is applied to the right angle to the plate". Fig.4 indicates that the primary velocity $u$ decreases with the increase of inclined angle $\alpha$. It is observed that the velocity in $x$-direction is highly increasing when the plate is properly vertical, i.e. when $\alpha=0$. Fig.5-9 elucidates the influence of pertinent parameters on the primary velocity $u$ for different values of $\beta_{e}, G_{r}, H_{a}, P_{r}$ and $D_{f}$ with the fixed value of $\alpha=\pi / 6$ (or, $\alpha=\pi / 3$ ). Fig.5 shows that $u$ increases with the increase of $\beta_{e}$. But from Fig.6 it is found in the main portion of the boundary layer that the velocity $u$ increases with the increase of Grashof number $G_{r}$. But the velocity is decreased with the increasing value of $H_{a}$ and $P_{r}$, which have shown in Fig.7 and Fig.8. It is observed from Fig.9 that $u$ has a minor increasing effect with the increase of $D_{f}$. 


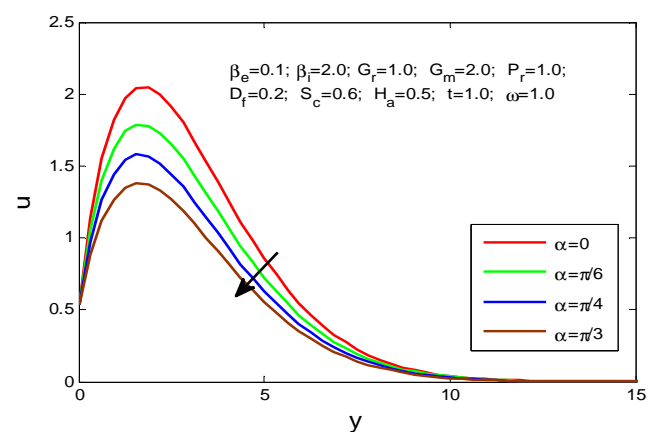

Fig.4: Primary velocity distribution $u$ for different values of inclined angle $\alpha$

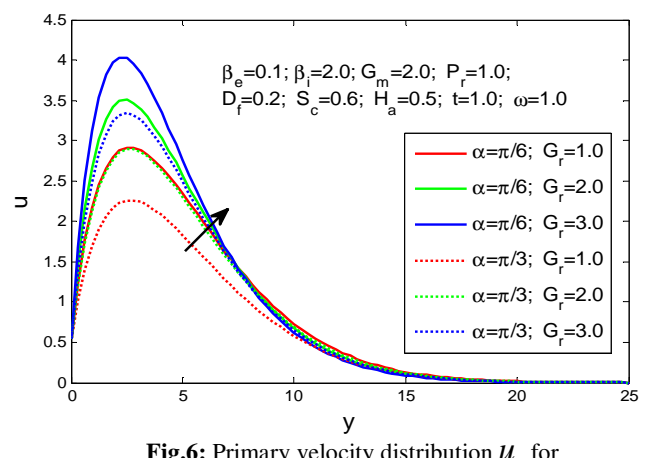

Fig.6: Primary velocity distribution $u$ for different values of Grashof number $G_{r}$

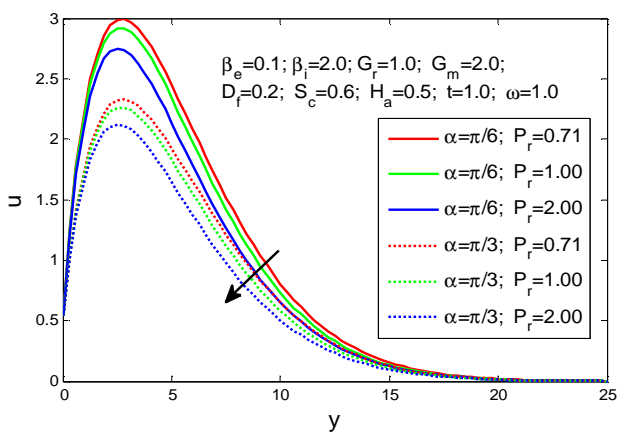

Fig.8: Primary velocity distribution $u$ for different values of Prandtl number $P_{r}$

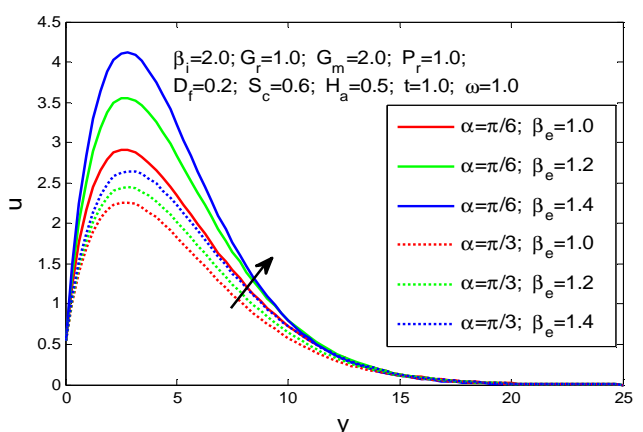

Fig.5: Primary velocity distribution $u$ for different values of Hall parameter $\beta_{e}$

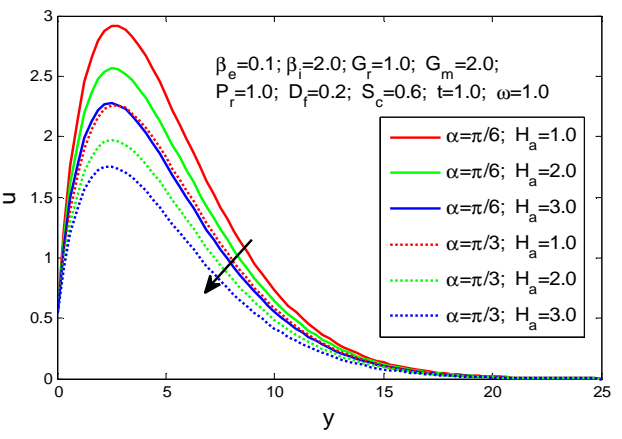

Fig.7: Primary velocity distribution $u$ for different values of Hartmann number $H_{a}$

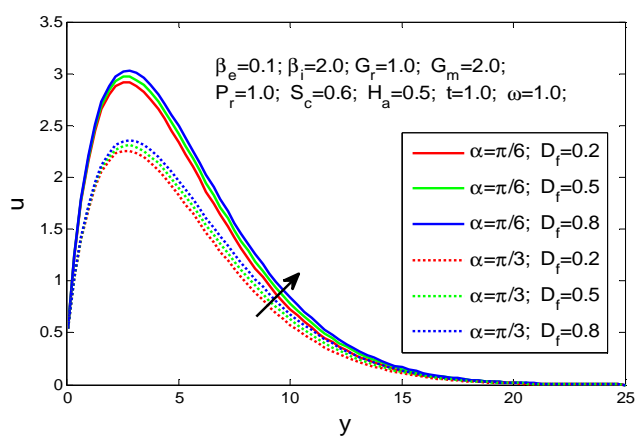

Fig.9: Primary velocity distribution $u$ for different values of Dufour number $D_{f}$

\section{Secondary Velocity Distributions}

Fig.10 exhibits the secondary velocity $w$ increases with the increase of inclined angle $\alpha$, but it has negative flow. Fig.11-15 indicate that the effect of parameters $\beta_{e}, \beta_{i}, P_{r}$ and $D_{f}$ on the secondary velocity $w$ with the fixed value of $\alpha=\pi / 6$ (or, $\pi / 3$ ). We observed that the velocities are very affected by the inclined angle $\alpha$. Fig.11 indicated that $w$ decreases with the increase of $\beta_{e}$. But from Figs.12-14 noticed that the velocity $w$ increases with the increase of $\beta_{i}, H_{a}, P_{r}$. The secondary velocity is decreased with the increasing value of $D_{f}$, which have shown in Fig.15.

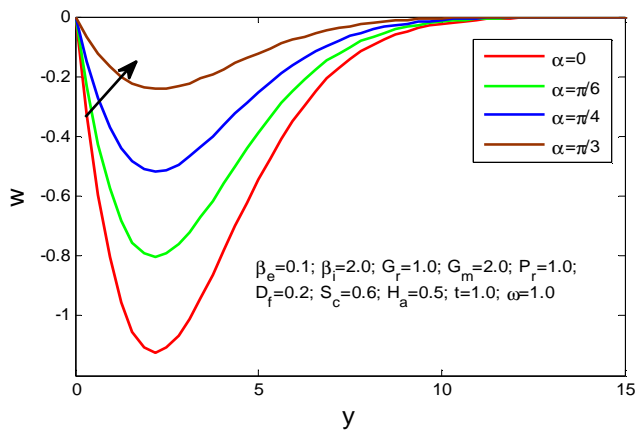

Fig.10: Secondary velocity distribution $w$ for different values of inclined angle $\alpha$

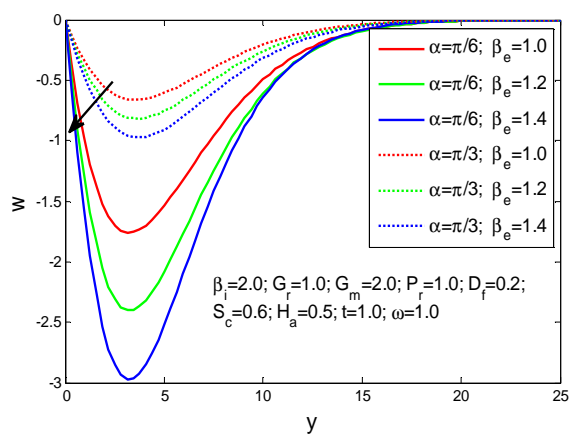

Fig.11: Secondary velocity distribution $w$ for different values of Hall parameter $\beta_{e}$ 


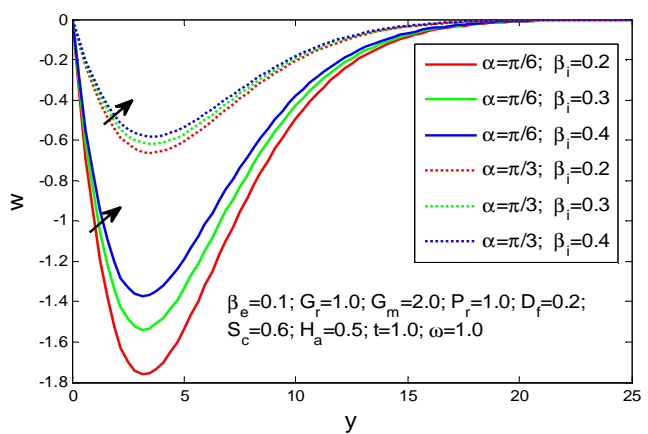

Fig.12: Secondary velocity distribution $w$ for different values of ion-slip parameter $\beta_{i}$

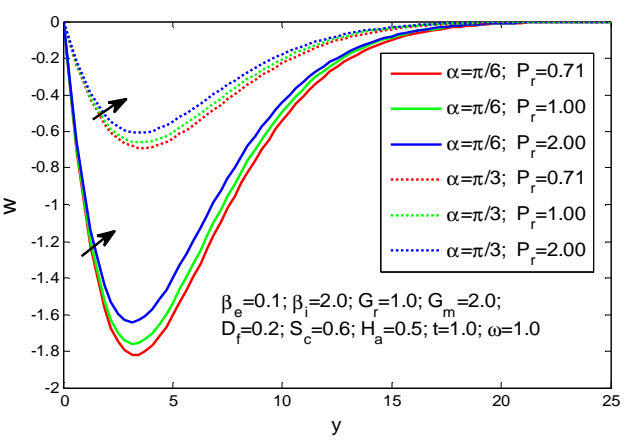

Fig.14: Secondary velocity distribution $w$ for different values of Prandtl number $P_{r}$

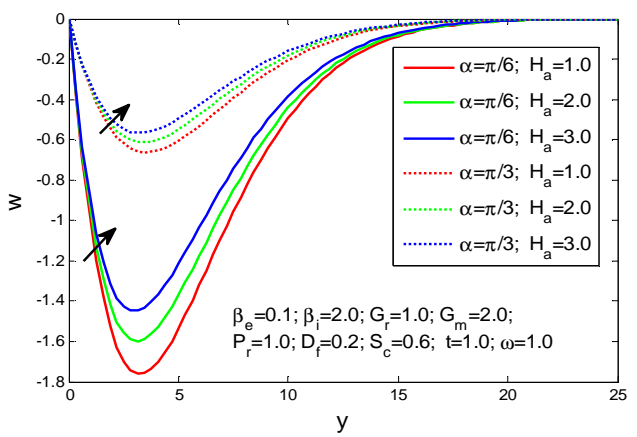

Fig.13: Secondary velocity distribution ' $w$ ' for different values of Hartmann number ' $H_{a}$ '

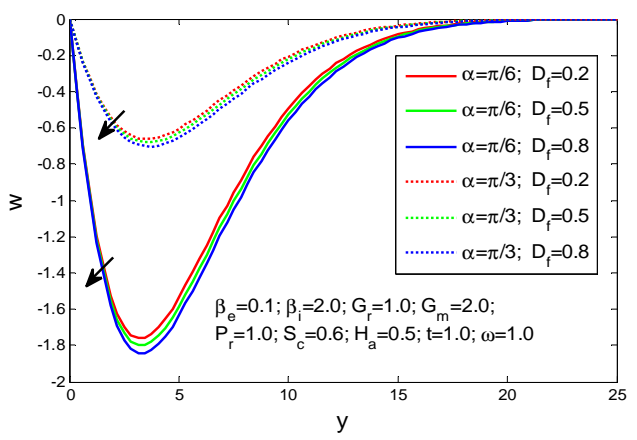

Fig.15: Secondary velocity distribution $w$ for different values of Dufour number $D_{f}$

\section{Temperature Distributions}

Fig.16 depicts the influence of the effective inclined angle $\alpha$ on the fluid temperature $\theta$. We have seen from the figure that the temperature is increased with the increase of $\alpha$. From Fig.17-19, it is investigated that the temperature is decreased with the increase of $\beta_{e}, G_{r}$ and $P_{r}$, whereas the temperature is increased with the increasing effect of $D_{f}$ and $S_{c}$, which have shown in Fig.20-21.

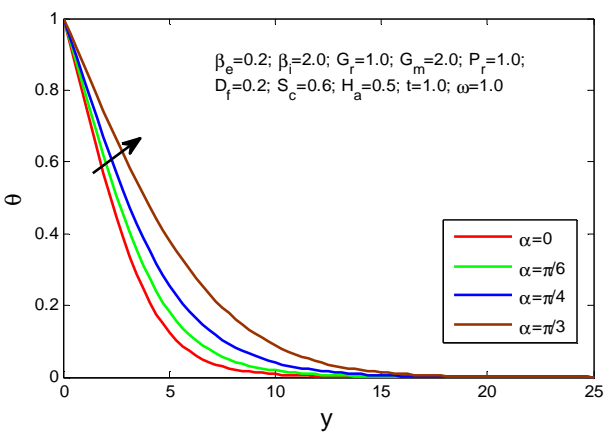

Fig.16: Temperature distribution $\theta$ for different values of inclined angle $\alpha$

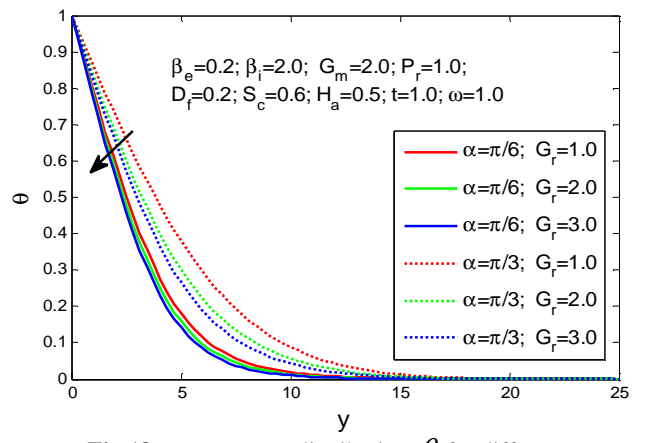

Fig.18: Temperature distribution $\theta$ for different values of thermal Grashof number $G_{r}$

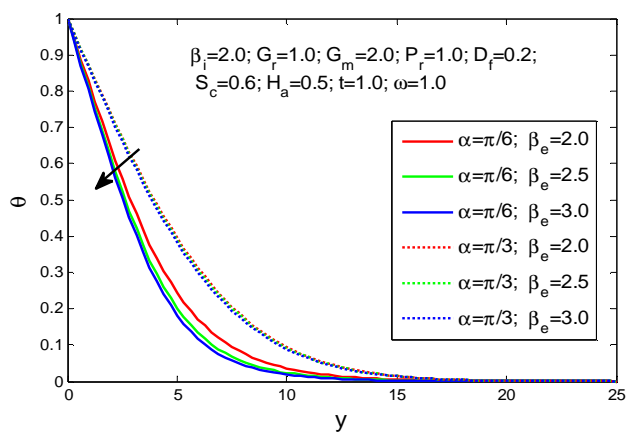

Fig.17: Temperature distribution $\theta$ for different values of Hall parameter $\beta_{e}$

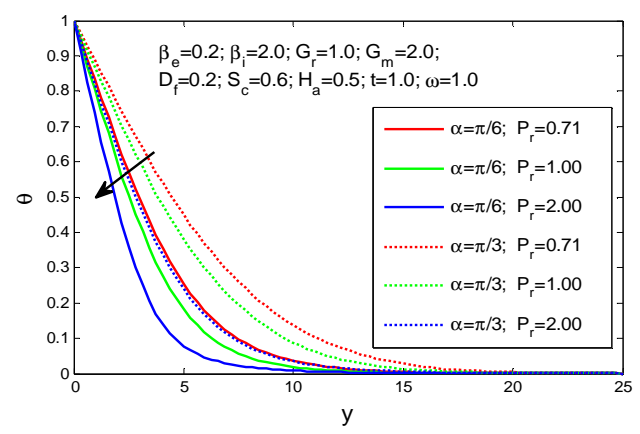

.19: Temperature distribution $\theta$ for different values of Prandtl number $P_{r}$ 


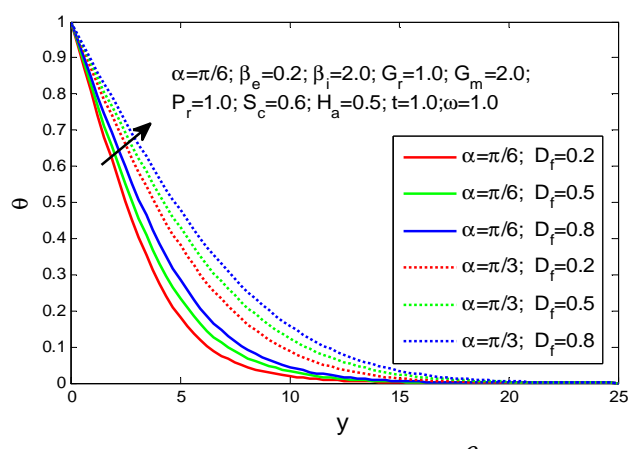

Fig.20: Temperature distribution $\theta$ for

different values of Dufour number $D_{f}$

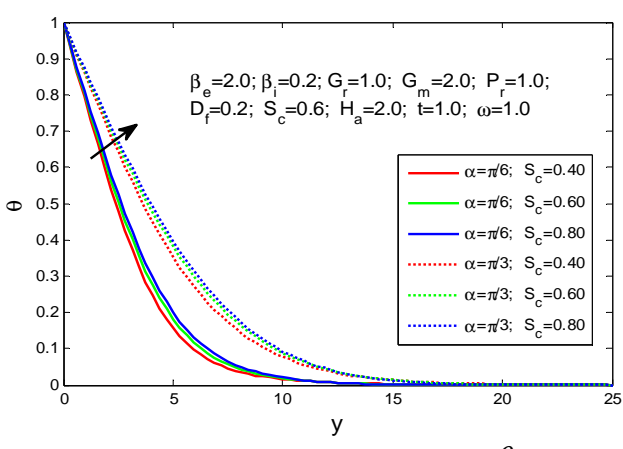

Fig.21: Temperature distribution $\theta$ for different values of Schmidt number $S_{c}$

\section{Concentration Distributions}

Fig. 22 represents the effect of inclined angle $\alpha$ on the fluid concentration $\phi$. It has indicated that the concentration is increased with the increase of $\alpha$. From Fig.23-25, we have analyzed for all cases that the concentration is decreased with the increase of $\beta_{e}, G_{m}$ and $S_{c}$.

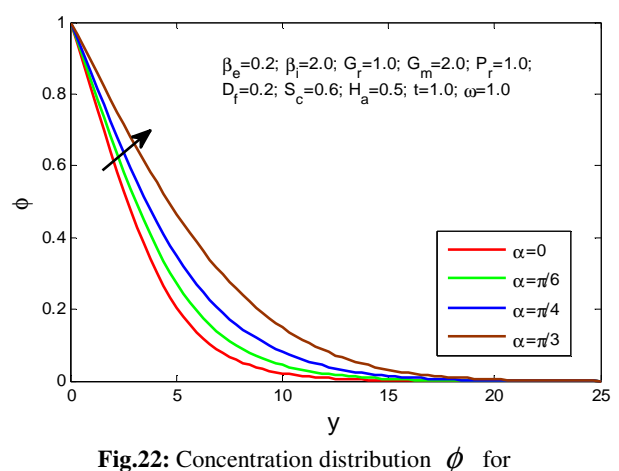

different values of inclined angle $\alpha$

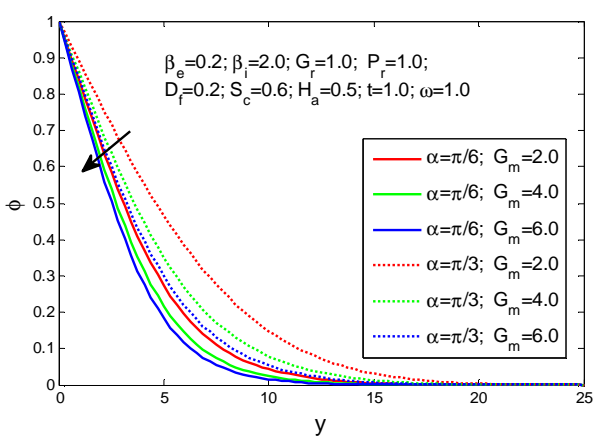

Fig.24: Concentration distribution $\phi$ for different values of mass Grashof number $G_{m}$

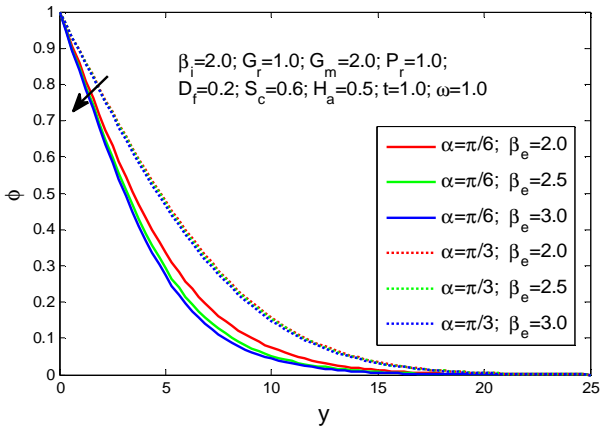

Fig.23: Concentration distribution $\phi$ for different values of Hall parameter $\beta_{e}$

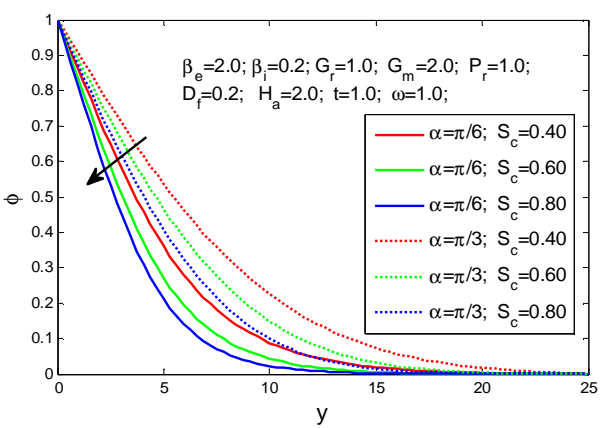

Fig.25: Concentration distribution $\phi$ for different values of Schmidt number $S_{c}$

\section{Local and Average Shear Stress}

Noted that the figures (a) and (b) of Fig.26-33 are represented the local shear stress $\left(\tau_{L}\right)$ and average shear stress $\left(\tau_{A}\right)$ respectively. Fig.26 depicts the effect of inclined angle $\alpha$ on the primary shear stress. We have seen that in both cases, the primary velocity gradient along $x$-direction of the plate decreases with the increase of $\alpha$. Fig.27-28, we have analyzed that the primary shear stress is increased with the increase of $\beta_{e}$ and $G_{r}$. But from Fig.29, we have seen that primary shear stress decreases with the increase of $H_{a}$. 


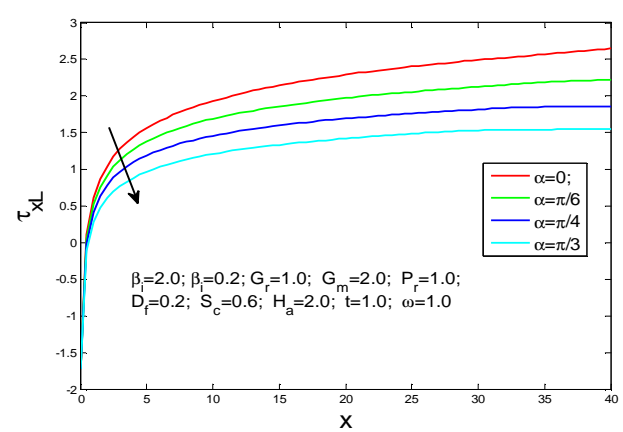

Fig.26(a): Local primary Shear stress $\tau_{x L}$ for different values of inclined angle $\alpha$

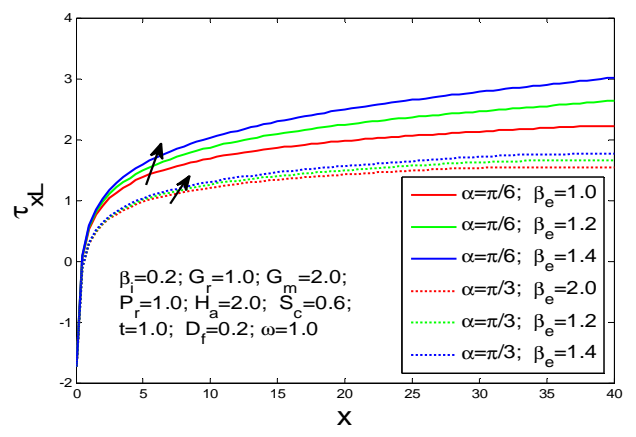

Fig.27(a): Local primary Shear stress $\tau_{x L}$ for different values of Hall parameter $\beta_{e}$

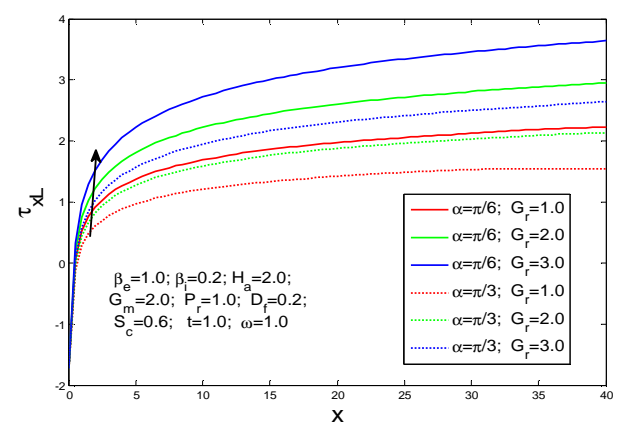

Fig.28(a): Local primary Shear stress $\tau_{x L}$ for different values of thermal Grashof number $G_{r}$

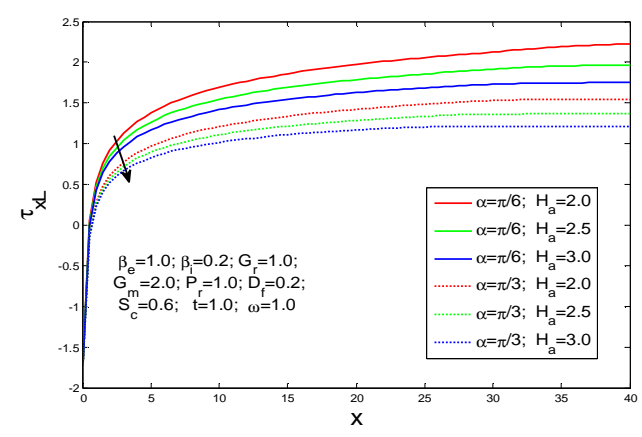

Fig.29(a): Local primary Shear stress $\tau_{x L}$ for different values of Hartmann number $H_{a}$

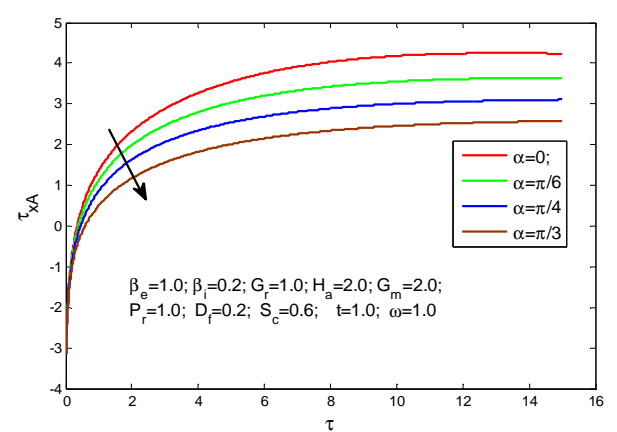

Fig.26(b): Average primary Shear stress $\tau_{x A}$ for different values of inclined angle $\alpha$

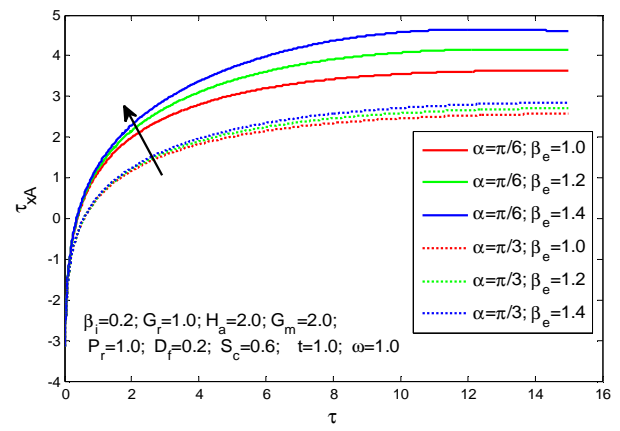

Fig.27(b): Average primary Shear stress $\tau_{x A}$ for different values of Hall parameter $\beta_{e}$

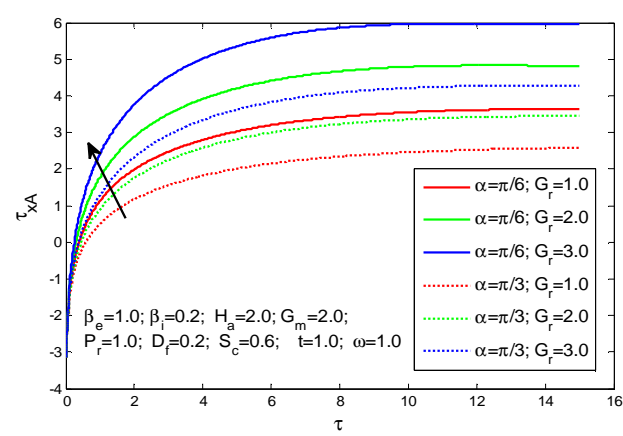

Fig.28(b): Average primary Shear stress $\tau_{x A}$ for different values thermal Grashof number $G_{r}$

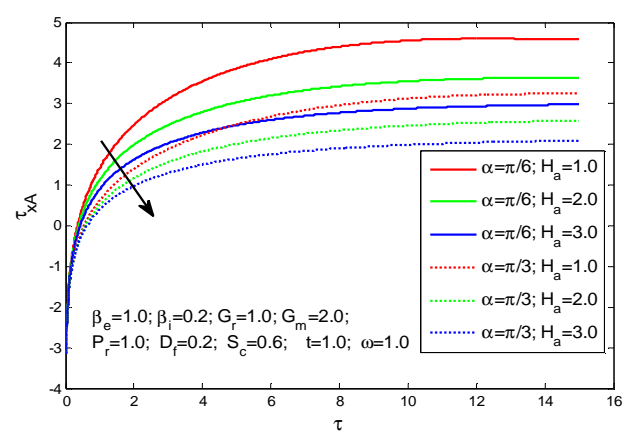

Fig.29(b): Local primary Shear stress $\tau_{x L}$ for different values of Hartmann number $H_{a}$

On the other hand, Fig.30 describes the effect of inclined angle $\alpha$ on the secondary shear stress. Here in both cases, the secondary velocity gradient along $x$-direction at the plate is increased with the increase of $\alpha$.Fig.31 has shown the secondary shear stress is increased with the increase of $\beta_{i}$. But Fig.32-33show, it has been decreasing effect with the increase of $\beta_{e}$ and $D_{f}$. It is observed that the effects of susceptive parameters on local shear stress, average shear stress and on velocity distributions have same. 


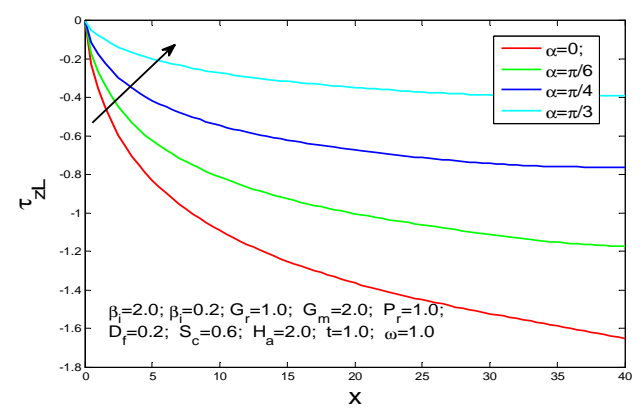

Fig.30(a): Local secondary Shear stress $\tau_{z L}$ for different values of inclined angle $\alpha$

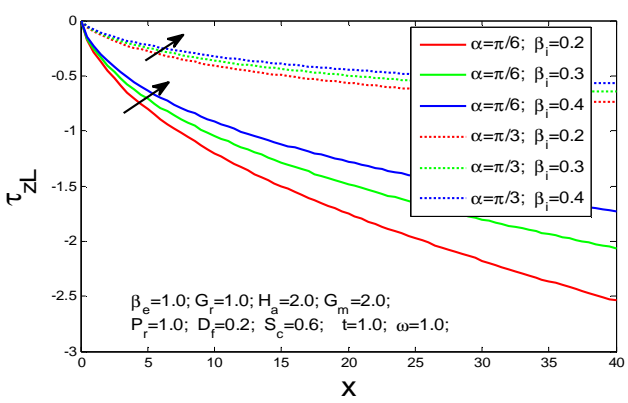

Fig.31(a): Local secondary Shear stress $\tau_{z L}$ for different values of ion-slip parameter $\beta_{i}$

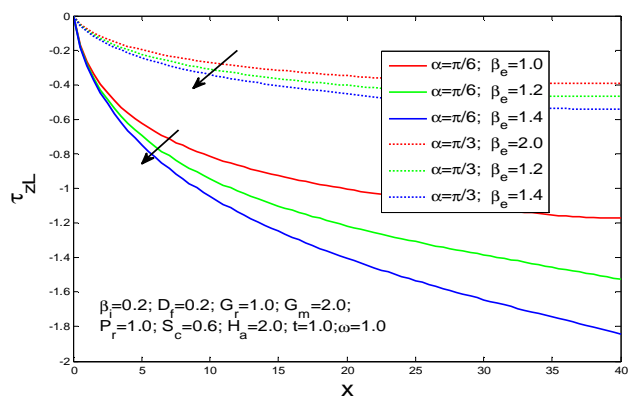

Fig.32(a): Local secondary Shear stress $\tau_{z L}$ for different values of Hall parameter $\beta_{e}$

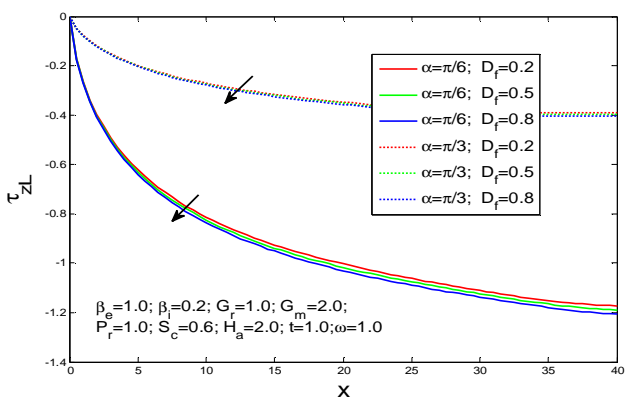

Fig.33(a): Local secondary Shear stress $\tau_{z L}$ for different values of Dufour number $D_{f}$

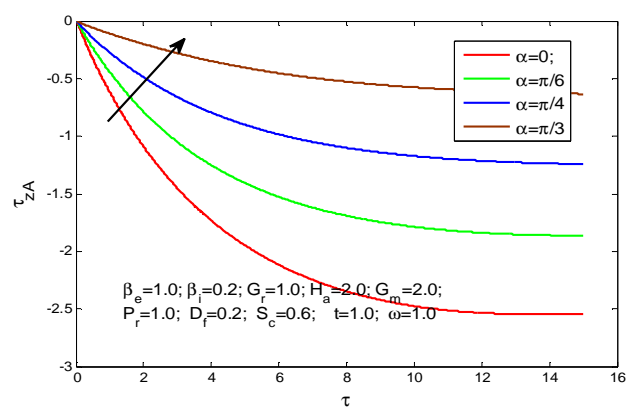

Fig.30(b): Average secondary Shear stress $\tau_{z A}$ For different values of inclined angle $\alpha$

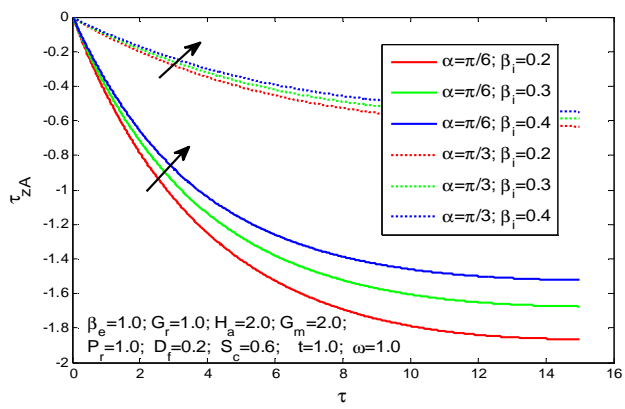

Fig.31(b): Average secondary Shear stress $\tau_{z A}$ for different values of ion-slip parameter $\beta_{i}$

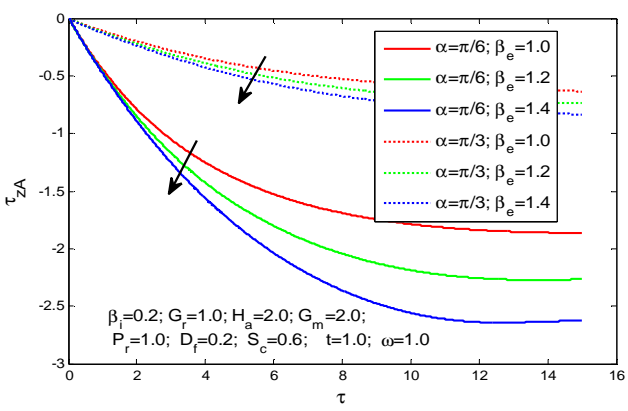

Fig.32(b): Average secondary Shear stress $\tau_{z A}$ for different values of Hall parameter $\beta_{e}$

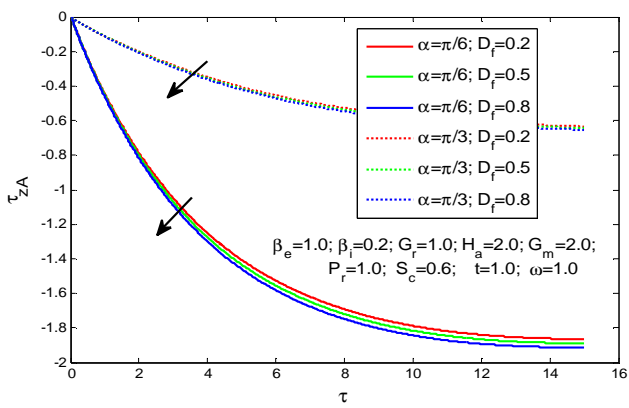

Fig.33(b): Average secondary Shear stress $\tau_{z A}$ for different values of Dufour number $D_{f}$

\section{Local and Average Nusselt Number, Sherwood Number:}

It is also noted that the figures (a) and (b) of Fig.34-35 are represented the local Nusselt number $\left(N u_{L}\right)$ and average Nusselt number $\left(N u_{A}\right)$. And figures (a) and (b) of Fig.36 are represented the local Sherwood number $\left(S h_{L}\right)$ and average Sherwood number $\left(S h_{A}\right)$ respectively. Fig.34 exhibits the effect of $D_{f}$ on the Nusselt number. It has seen that the concentration gradient at the wall decreases with the increase of $D_{f}$. But Fig.35 shows that the Nusselt number is increased with the increase of $P_{r}$. On the other hand, the Sherwood number is increased with the increasing effect of $S_{c}$, which have shown in Fig.36. 


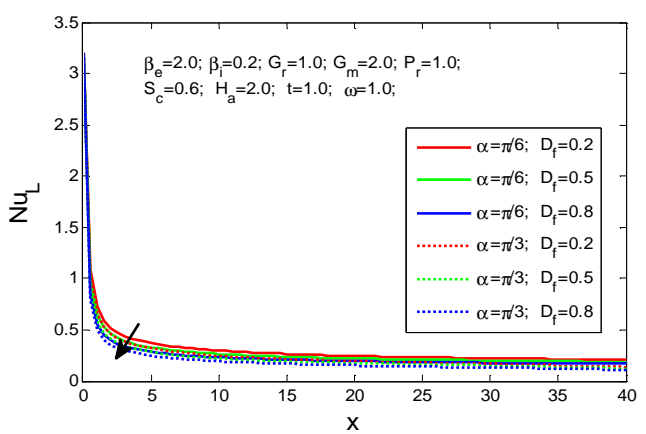

Fig.34(a): Local Nusselt number $N u_{L}$ for different values of Dufour number $D_{f}$

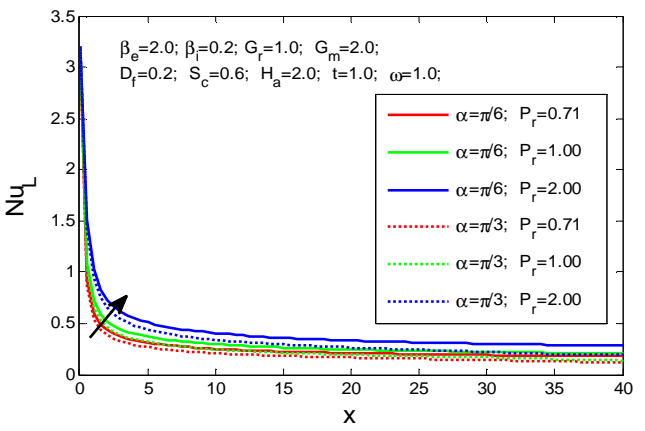

Fig.35(a): Local Nusselt number $N u_{L}$ for different values of Prandtl number $P_{r}$

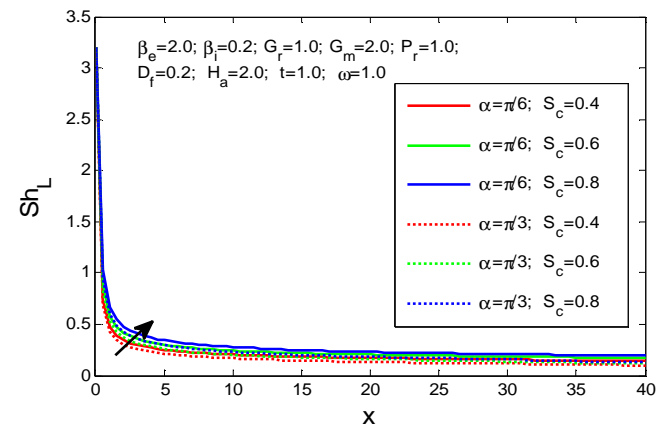

Fig.36(a): Local Sherwood number $S h_{L}$ for different values of Schmidt number $S_{c}$

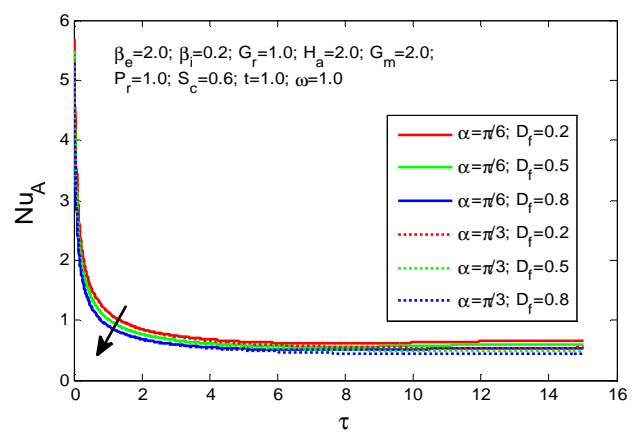

Fig.34(b): Average Nusselt number $N u_{A}$ for different values of Dufour number $D_{f}$

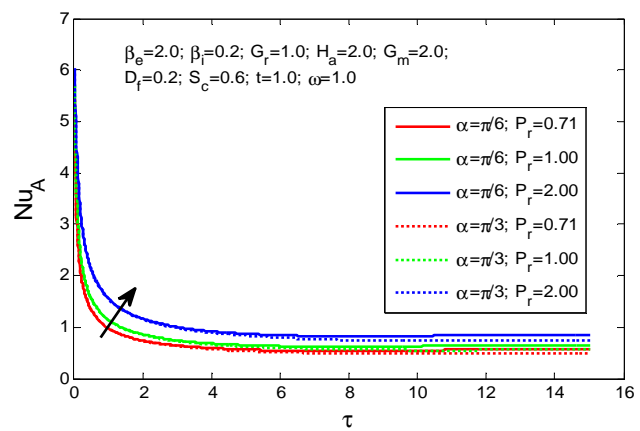

Fig.35(b):Average Nusselt number $N u_{A}$ for different values of Prandtl number $P_{r}$

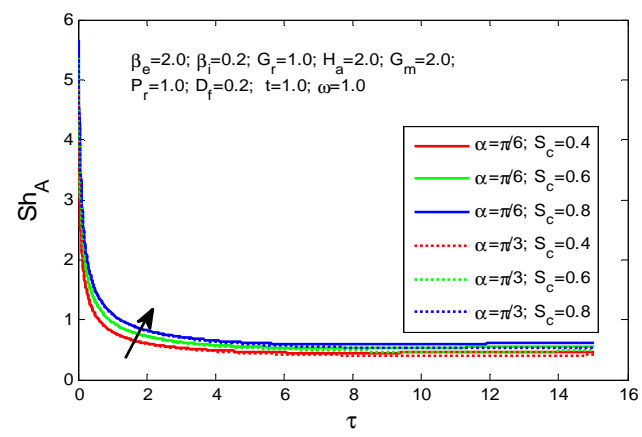

Fig.36(b): Average Sherwood number $S h_{A}$ for different values of Schmidt number $S_{c}$

\section{Conclusion}

The angle of inclination, Hall and ion slip current effects on the unsteady MHD fluid flow without change of reference frame has been analyzed. Some important findings of our study are as below:

Primary velocity $u$ rises with the increase of $\beta_{e}, G_{r}$ and $D_{f}$ while it decreases with the increase of $\alpha, H_{a}$ and $P_{r}$.

$>$ Secondary velocity $w$ increases with the increase of $\alpha, \beta_{i}, H_{a}$ and $P_{r}$, it has a decreasing effect with the increase of $\beta_{e}$ and $D_{f}$.

$>$ Temperature $\theta$ is improved with the increase of $\alpha, S_{c}$ and $S_{c}$, whereas it has a decreasing effect with the increase of $\beta_{e}$, $G_{r}$ and $P_{r}$.

Concentration $\phi$ is increased with the increase of $\alpha$, whereas it has been decreasing effect with the increase of $\beta_{e}, G_{m}$ and $S_{c}$.

\section{Nomenclature}

q : Fluid velocity vector

J : Current density vector

$u$ : Velocity components along $x$ axis

$v$ : Velocity components along $y$ -
B : Magnetic field vector

$g$ : Acceleration due to the gravity

$U_{0}$ : Constant uniform velocity

$t$ : Time

$\Delta t:$ Time increment
$T$ : Temperature of the fluid

$T_{w}:$ Constant temperature near the plate

$T_{\infty}$ : Temperature outside of the boundary layer

$C$ : Concentration in the fluid 
axis

$w$ : Velocity components along $z$ axis

$\beta$ : Volumetric coefficient of thermal expansion

$\beta^{*}$ : Volumetric coefficient of mass expansion

$B_{0}$ : Constant magnetic induction along $y$-directions

$\alpha$ : Inclined angle

$\omega$ : Acceleration frequency

$\omega_{e}:$ Cyclotron frequency

$\rho$ : Density of the fluid

$\sigma \quad$ : Conductivity of the fluid

$\tau_{e}$ : Electron collision time

$c_{p}$ : Specific heat at the constant pressure

$k_{T}$ : Thermal diffusion ratio

$v$ : Kinematic viscosity of the fluid $\tau$ : Maximum time

$c_{s}$ : Concentration susceptibility

$\sigma^{\prime}$ : Electric conductivity

$\mu$ : Coefficient of viscosity of the fluid

$\mu_{e}$ : Magnetic permeability

$\beta_{e}$ : Hall parameter

$\beta_{i}$ : Ion-slip parameter

$u^{*}$ : Dimensionless velocity

component in $x$-axis

$v^{*}$ : Dimensionless velocity

component in $y$-axis

$t^{*} \quad$ : Dimensionless time

$\omega^{*}$ : Dimensionless acceleration parameter

$\theta$ : Dimensionless temperature

$\phi \quad$ : Dimensionless fluid

concentration

$D_{m}$ : Mass diffusion coefficient
$C_{w}$ : Constant concentration near the plate

$C_{\infty}$ : Concentration outside of the boundary layer

$H_{a}$ : Hartmann number

$G_{r}$ : Thermal Grashof number

$G_{m}:$ Solutal Grashof number

$P_{r}$ : Prandtl number

$S_{c}:$ Schmidt number

$D_{f}:$ Dufour number

$\tau_{x L}$ : Local primary shear stress

$\tau_{z L}$ : Local secondary shear stress

$N u_{L}$ : Local Nusselt number

$S h_{L}$ : Local Sherwood number

$\tau_{x A}:$ Average primary shear stress

$\tau_{z A}:$ Average primary shear stress

$N u_{A}$ : Average Nusselt number

$S h_{A}:$ Average Sherwood number

\section{References}

1. Abel, S. and Veena, P. H. Visco-Elastic Fluid Flow and Heat Transfer in Porous Medium over a Stretching Sheet, International Journal of Non-Linear Mechanics, Vol. 33, pp. 531-540 (1998).

2. Abernathy, F.H. Flow over an inclined plate, Journal of Fluids Engineering, Vol. 84(3),pp. 380-388 (1962).

3. Ajay K. S. MHD free convection and mass transfer flow with Hall current, viscous dissipation, joule heating and thermal diffusion, Indian journal of pure and applied physics, vol.41, pp-24-35 (2003).

4. Angirasa, D. Peterson, G.P. Natural Convection Heat Transform from an Isothermal Vertical Surface to a Fluid Saturated Thermally Stratified Porous Medium, Int. J. Heat Mass Transfer, Vol. 14(8), pp. 4329-4335 (1997).

5. Basant K. J. and Peter B. M. Hall current and ion-slip effects on free convection flow in a vertical micro channel with an induced magnetic field", Heat transfer Asian Research, 10.1002/htj.21569 (2019).

6. Beg, O. A. Sim, J. L. Zueco, R. Bhargava, R. Numerical study of magneto-hydrodynamic viscous plasma flow in rotating porous media with Hall currents and inclined magnetic field influence, Communications in Nonlinear Science and Numerical Simulation, Vol.15, pp.345-359 (2010).

7. Bhpendra K. S., Abhay K. J., Chaudhary R.C. Hall effect on MHD mixed convective flow of a viscous incompressible fluid past a vertical porous plate immersed in porous medium with heat source/sink, Romanian Journal of Physics, vol.52,pp.5-7 (2007).

8. Daniel S. and Daniel, Y. S.(2013). Convective flow two immiscible fluids and heat transfer with porous along an inclined channel with pressure gradient, International Journal of Engineering and Science, vol. 2, no. 4, pp. 12-18.

9. Eraslan, A.H. Temperature distributions in MHD channels with hall effect, AIAAJ, Vol.7, pp.186-188 (1969).

10. Gupta PS and Gupta, A. S. "Heat and Mass Transfer on a Stretching Sheet with Suction or Blowing, Canadian Journal of Chemical Engineering, Vol.55, pp. 744-746 (1997).

11. Javeri, V. Combined influence of Hall effect, ion slip, viscous dissipation and Joule heating on MHD heat transfer in a channel, Heat and Mass Transfer, Vol.8, pp.193-202 (1975).

12. Jyotsna R. P., Gouranga C. D. and Suprava S. Radiation and mass transfer effects on MHD flow through porous medium past an exponentially accelerated inclined plate with variable temperature, Ain Shams Engineering Journal, Vol.8, pp.67-75 (2017).

13. Kumar, B.V.R. Singh, P. Effect of Thermal Stratification on Free Convection in a Fluid Saturated Porous Enclosure, Numer. Heat Transfer, Vol. 34, pp. 343-356 (1999).

14. Navnet Kumar Singh, Vinod Kumar and Gaurav Kumar Sharma, The effect of inclined magnetic field on unsteady flow past on moving vertical plate with variable temperature", IJLTEMAS, Vol.5, Issu. 2 (2016).

15. Opanuga, A.A. Agboola, O.O. Okagbue, H. I. Olanrewaju, A.M. Hall Current and Ion-Slip Effects on the Entropy Generation of Couple Stress Fluid with Velocity Slip and Temperature Jump, International journal of Mechanics, Vol.12 (2018).

16. Prasada, V. Sivaprasad R.and Rao, U.R. Hall effects on hydromagnetic channel flow under an inclind magnetic field, Indian natn.sci.Acad. 52,A ,No.3, pp.573-583 (1986).

17. Rajput U.S. and Gupt, N.K. Dafour effect on unsteady free convection MHD flow past an exponentially accelerated plate through porous media with variable temperature and constant mass diffusion in an inclined magnetic field, IRJET, Vol.3,Issu.8 (2016).

18. Ram, P. C. The effect of Hall and ion slip current on free convection heat generating flow in a rotating fluid, Int.J. Energy Res. Vol. 19(5), pp.371-376 (1995).

19. Rishab Richard Hanvey, Rajeev Kumar Khare, Ajit PaulMHD flow of incompressible fluid through parallel plates in inclind magnetic field having porous medium with heat and mass transfer, IJSIMR,Vol.5,Issu.4, pp.18-22 (2017).

20. Sato, H. The Hall Effect in the Viscous Flow of Ionized Gas Between two Parallel Plates under Transverse Magnetic Field, $J$. Phys. Soc. Jpn. Vol.16, pp.1427-1433 (1961).

21. Seth, G. S. Raj Nandkeolyar, Mahto N. and Singh, S. K. MHD Couette Flow in a Rotating System in the Presence of an Inclined Magnetic Field, Applied Mathematical Sciences, Vol. 3, no. 59, pp.2919 - 2932 (2009). 
22. Seth, G.S. Raj Nandkeolyer, Ansary, M.S. Hartmann flow in a rotating system in the present of inclined magnetic field with hall effects, Tamkang journal of science and engineering,Vol.13, No.3, pp.243-252. (2010).

23. Seth, G.S. Mandal P.K. and Chamkha A.J. MHD free convection flow past an impulsively moving vertical plate with ramped heat flux through porous medium in the presence of inclined magnetic field, FHMT,7,23 (2016).

24. Suman Agarwalla and Nazibuddin Ahmed, MHD mass transfer flow past an inclined plate with variable temperature and plate velocity embedded in a porous medium, Heat Transfer, Vol.47, Issu 1, pp.27-41(2018).

25. Veera Krishna, M. Ameer Ahmad N. and Ali J. Chamkha, Hall and ion slip effects on unsteady MHD free convective rotating flow through a saturated porous medium over an exponential accelerated plate, Alexandria Engineering Journal, Vol.59, Isse.2, pp. 565-577 (2020). 
Figures

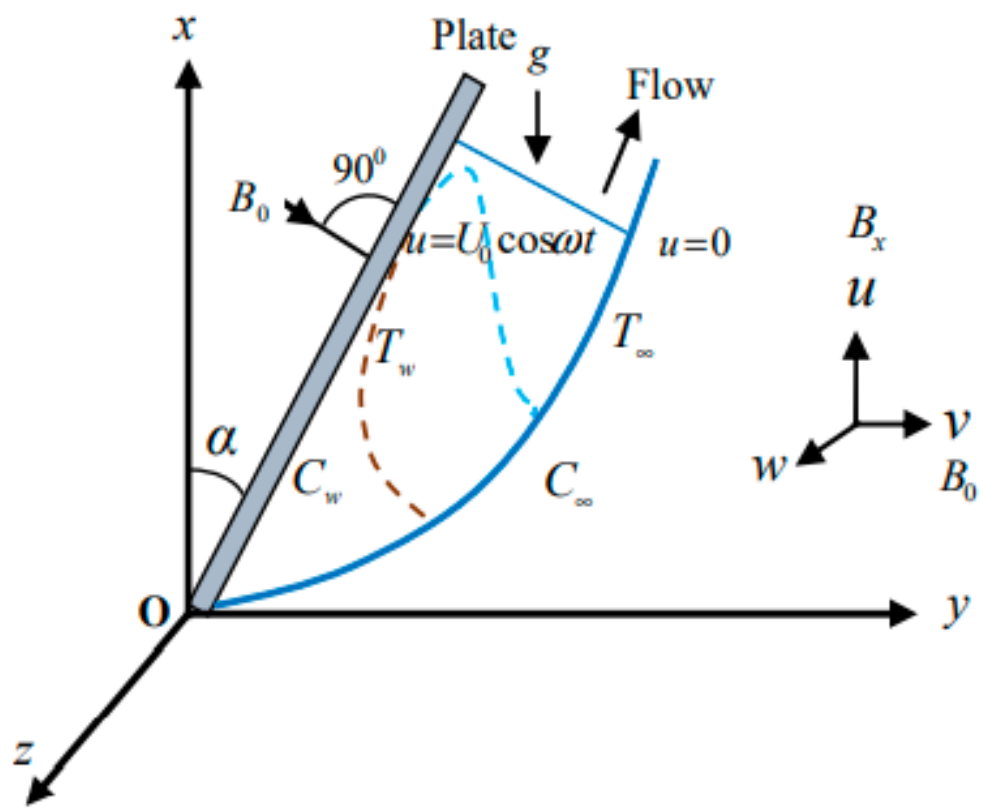

Figure 1

Please see the Manuscript PDF file for the complete figure caption

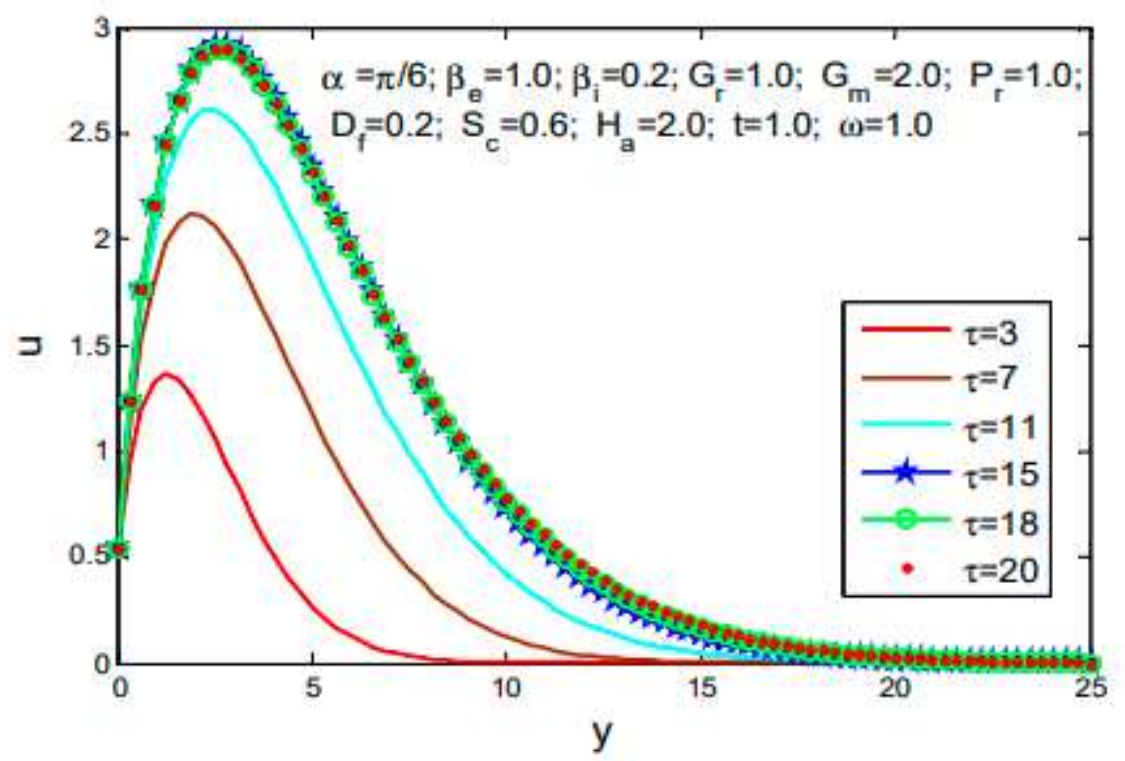

Figure 2

Please see the Manuscript PDF file for the complete figure caption 


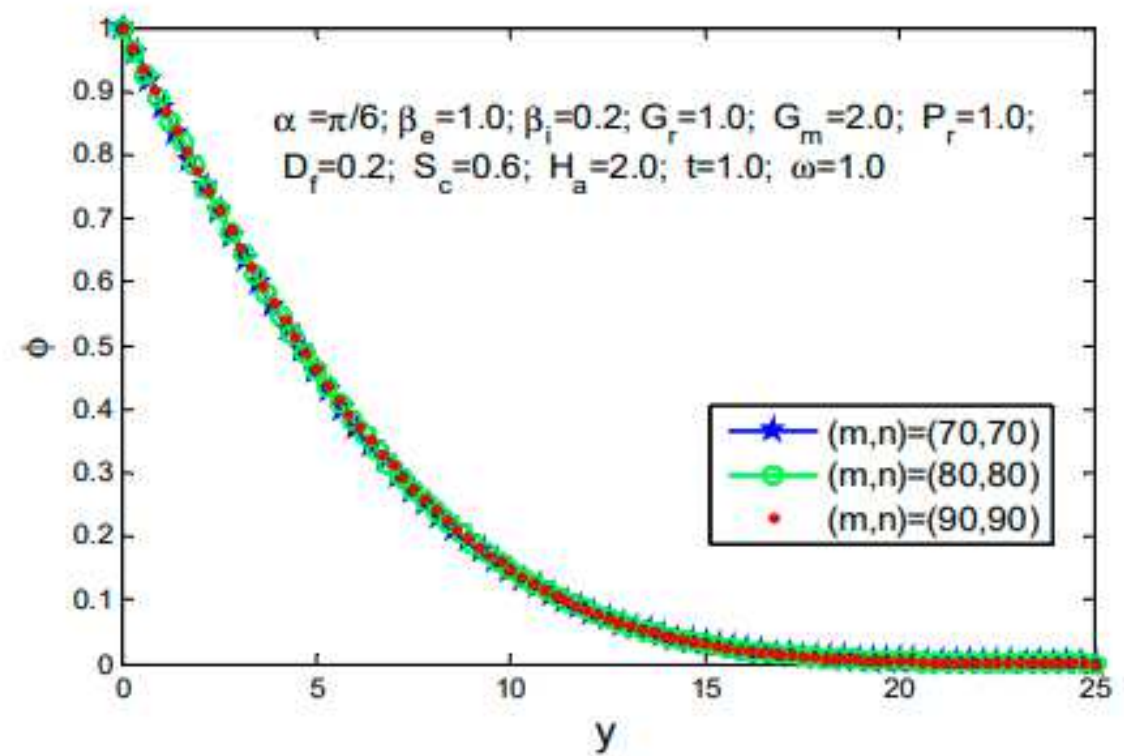

Figure 3

Please see the Manuscript PDF file for the complete figure caption

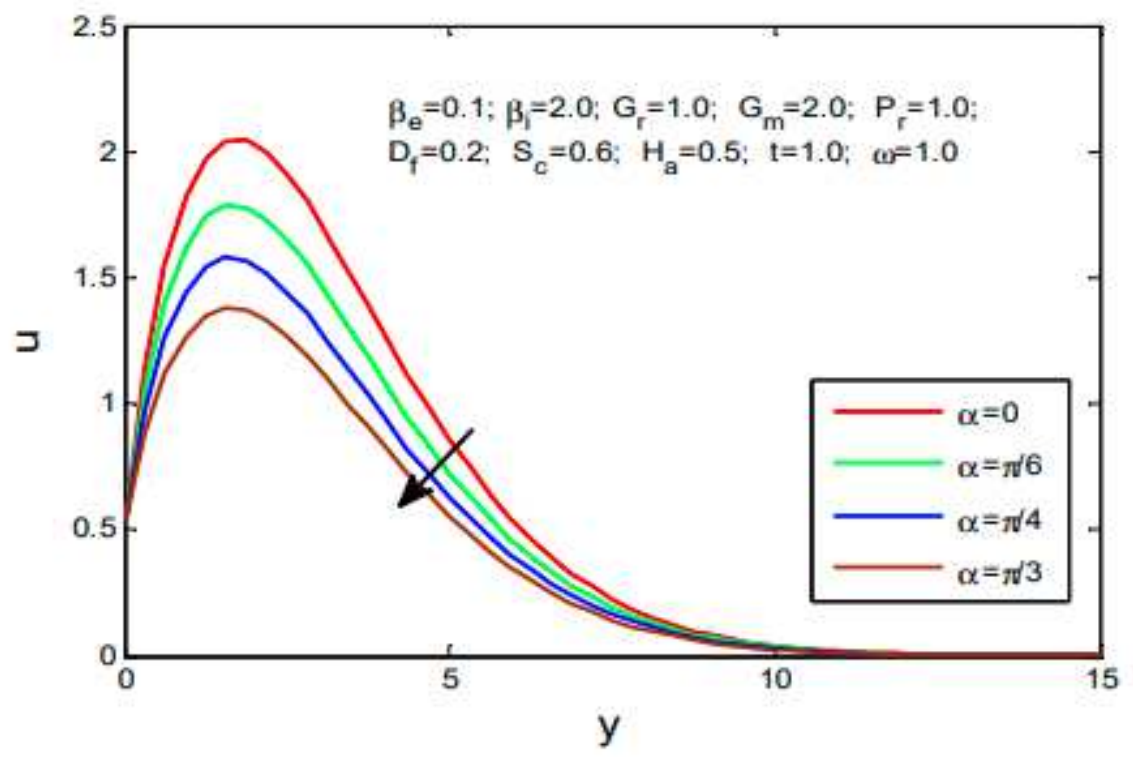

Figure 4

Please see the Manuscript PDF file for the complete figure caption 


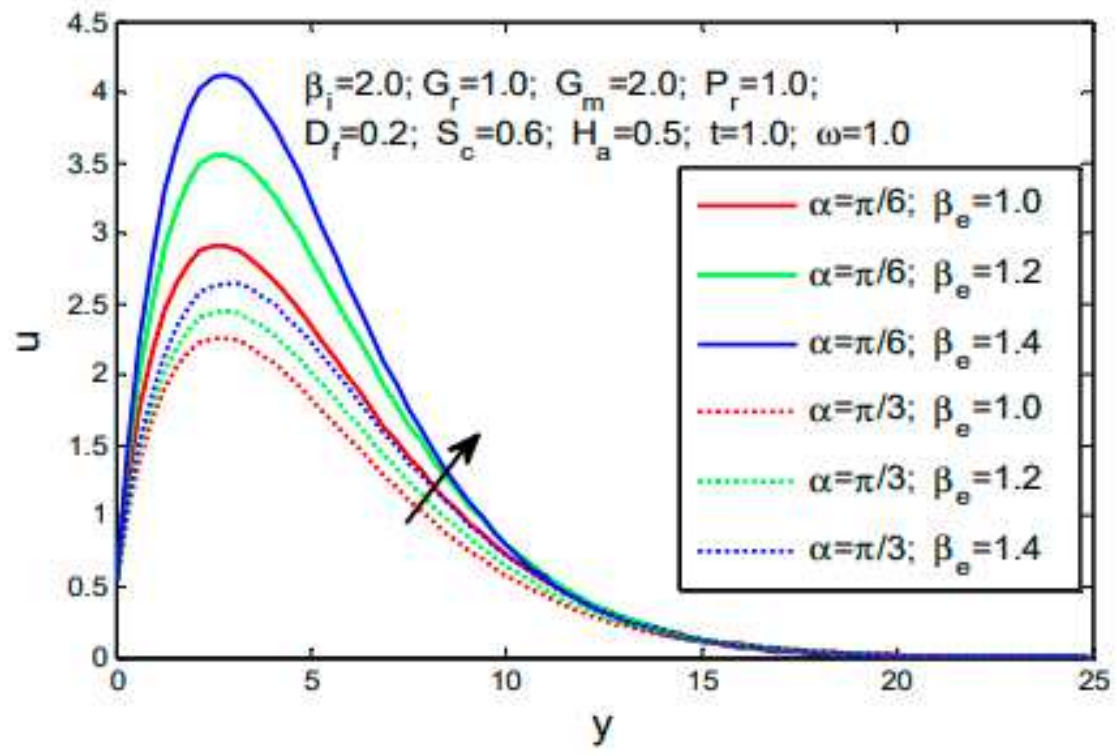

Figure 5

Please see the Manuscript PDF file for the complete figure caption

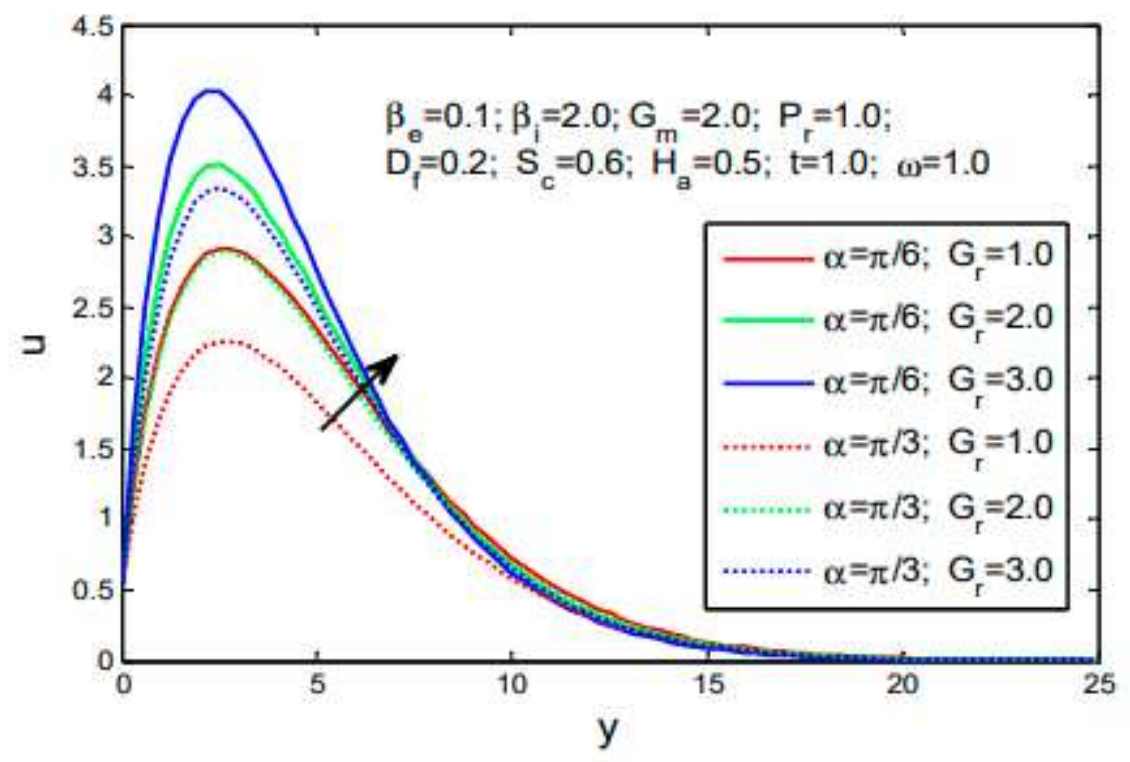

Figure 6

Please see the Manuscript PDF file for the complete figure caption 


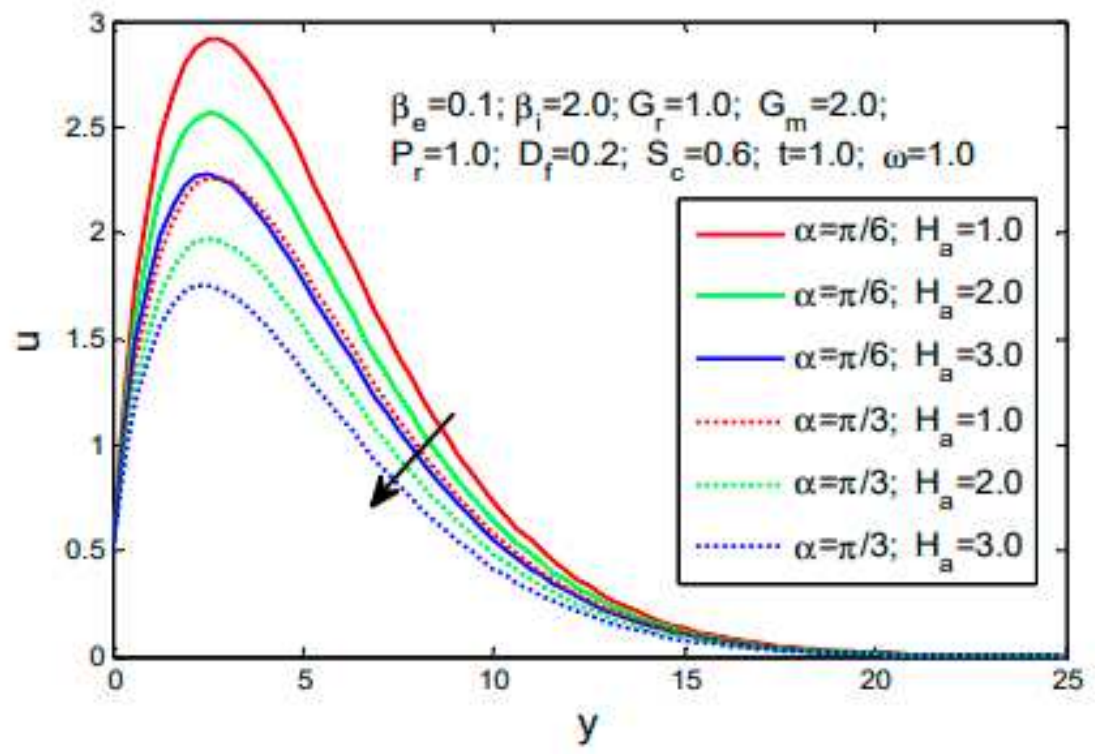

Figure 7

Please see the Manuscript PDF file for the complete figure caption

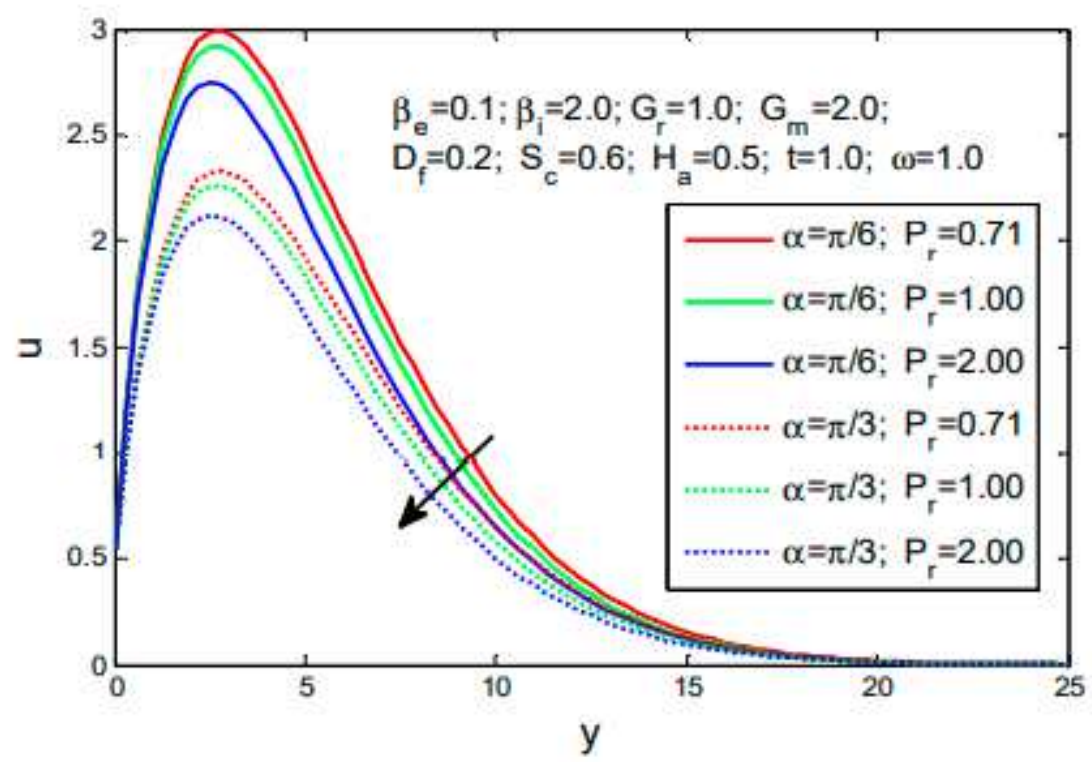

Figure 8

Please see the Manuscript PDF file for the complete figure caption 


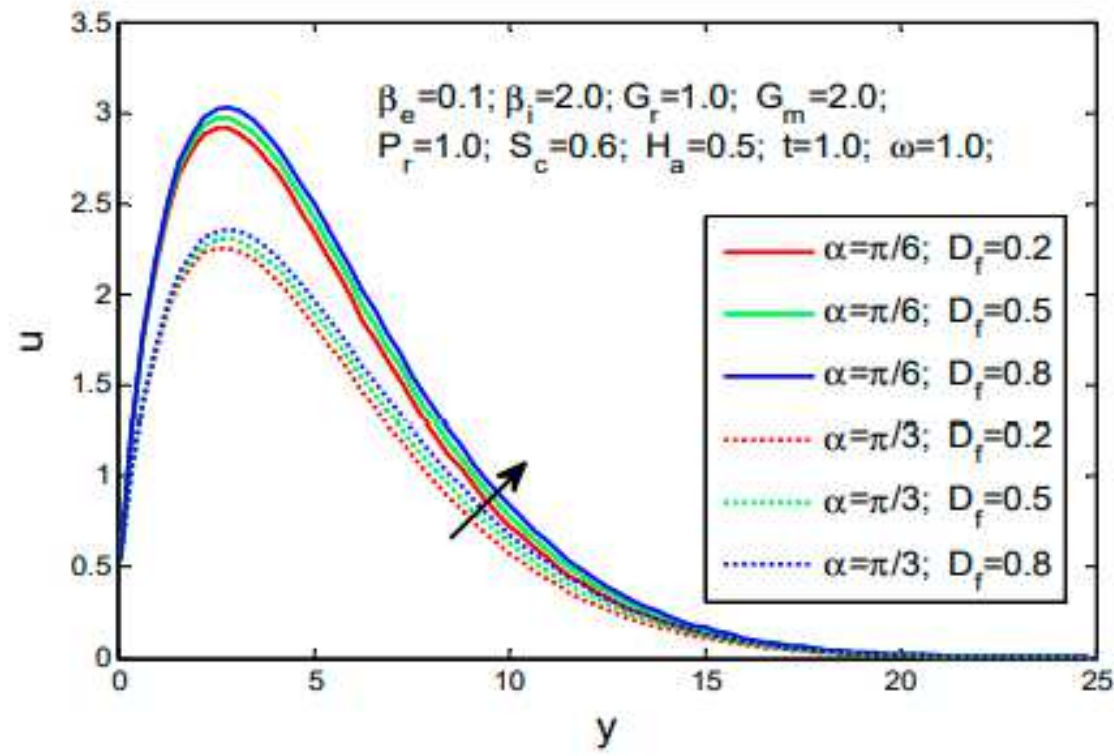

Figure 9

Please see the Manuscript PDF file for the complete figure caption

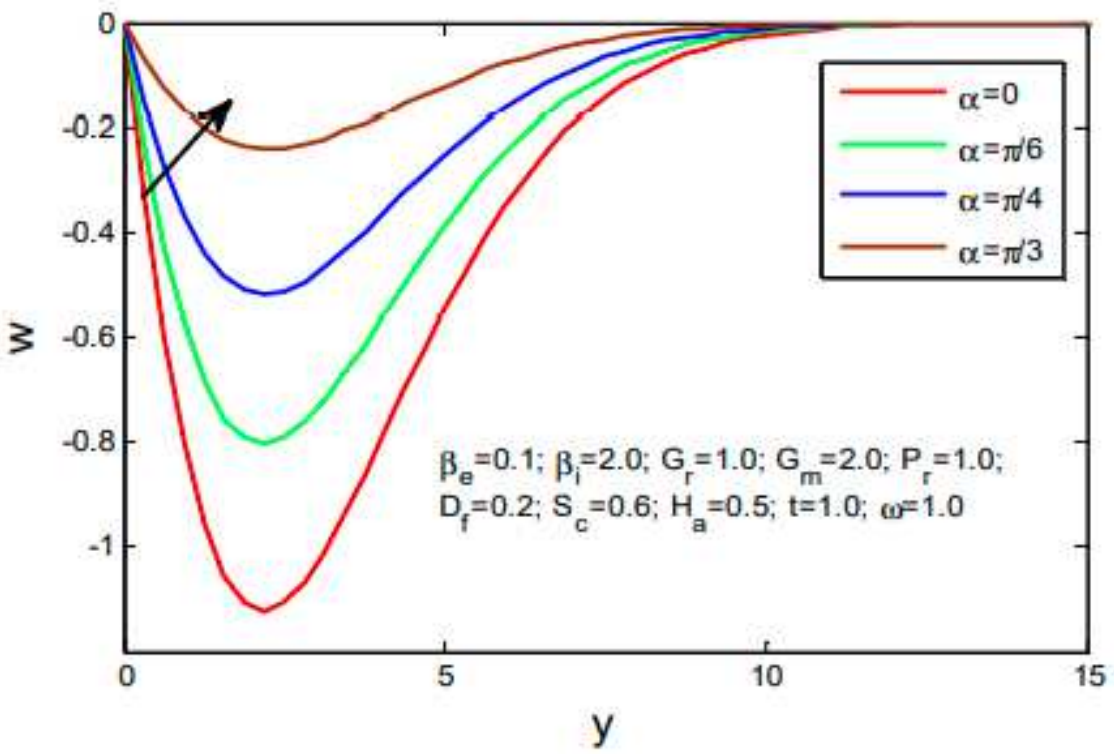

Figure 10

Please see the Manuscript PDF file for the complete figure caption 


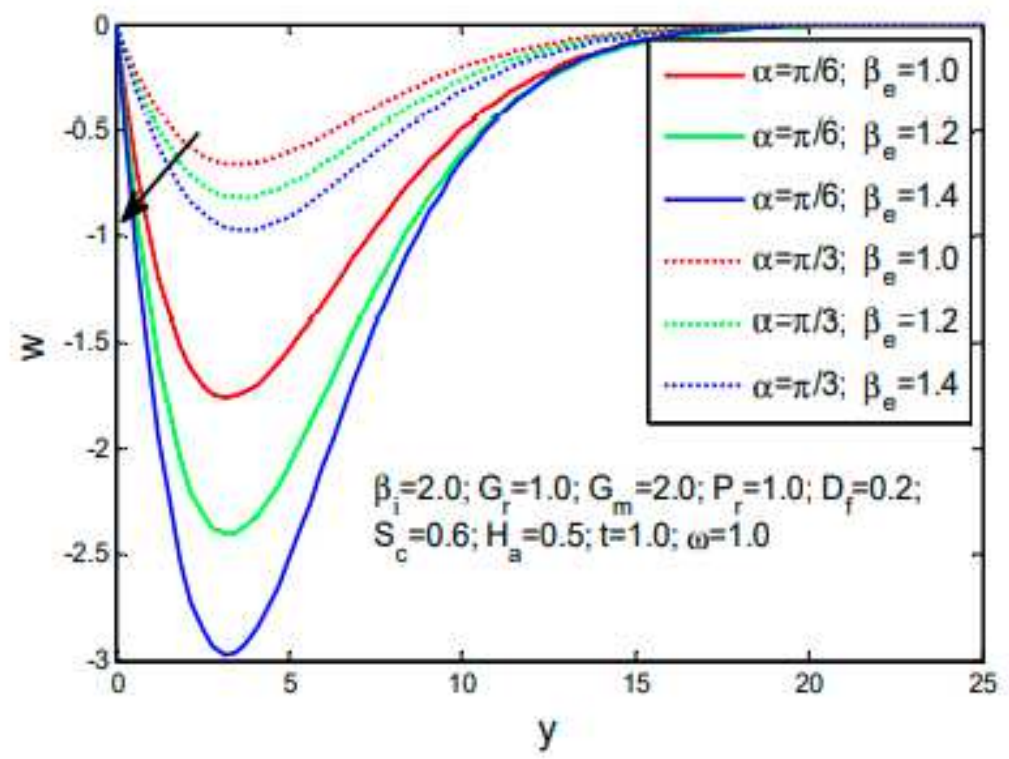

Figure 11

Please see the Manuscript PDF file for the complete figure caption

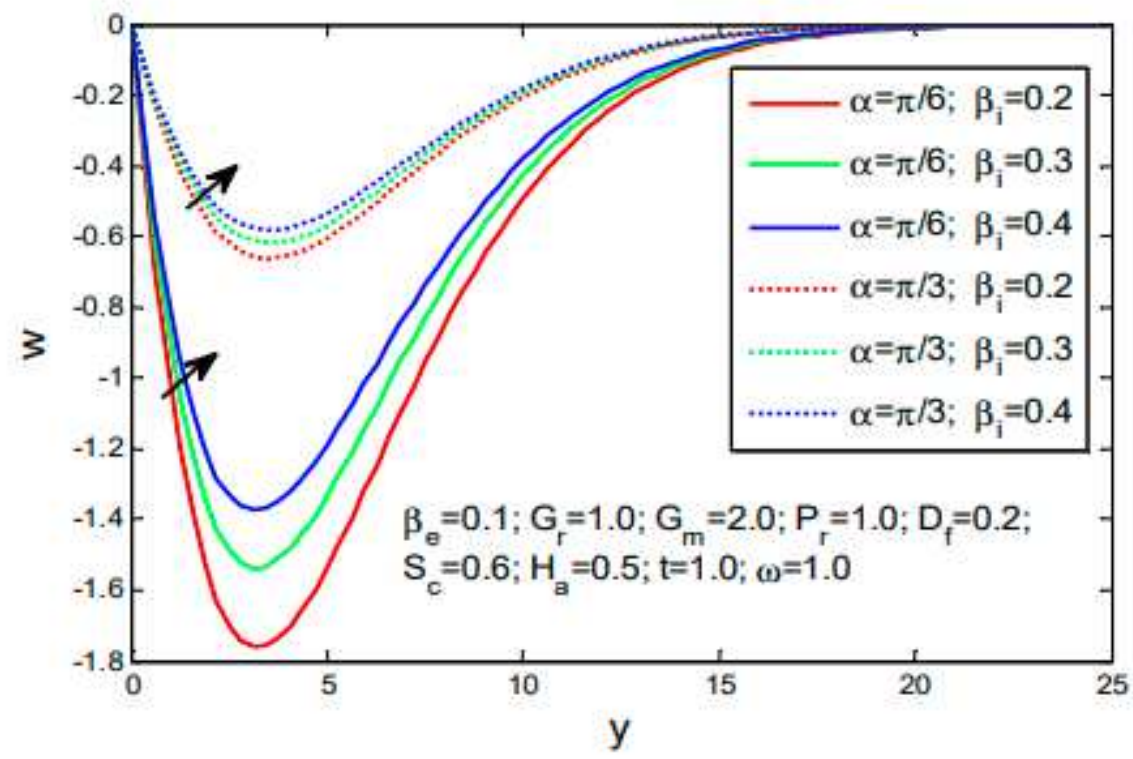

Figure 12

Please see the Manuscript PDF file for the complete figure caption 


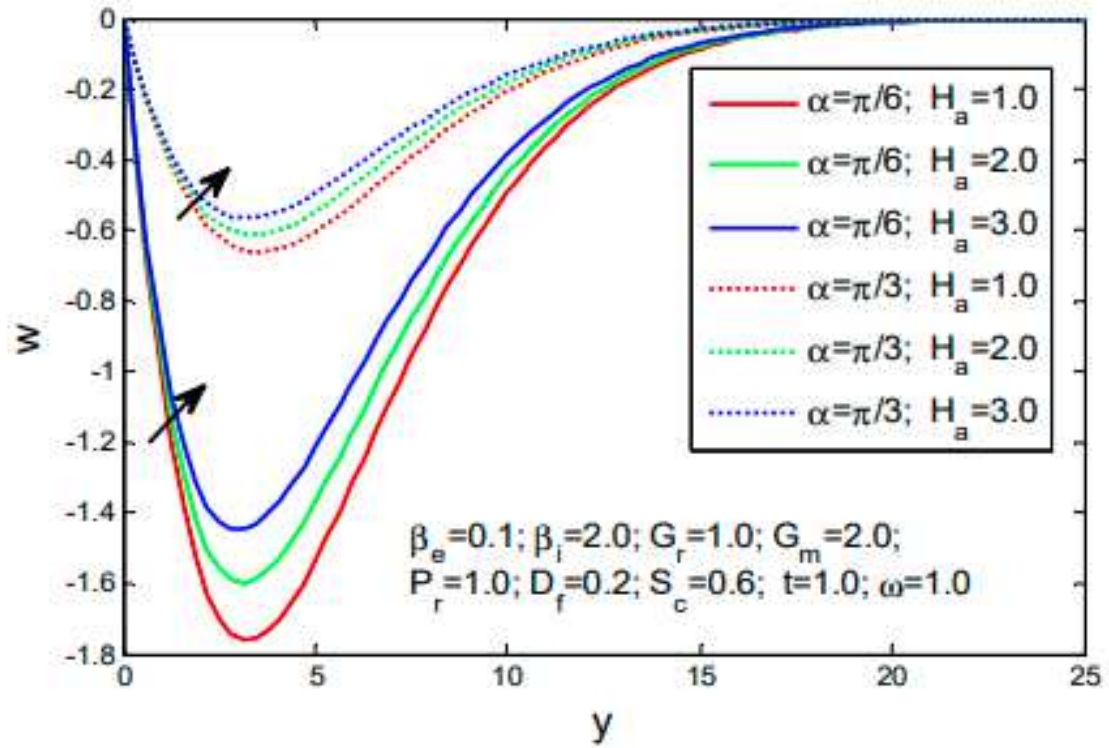

Figure 13

Please see the Manuscript PDF file for the complete figure caption

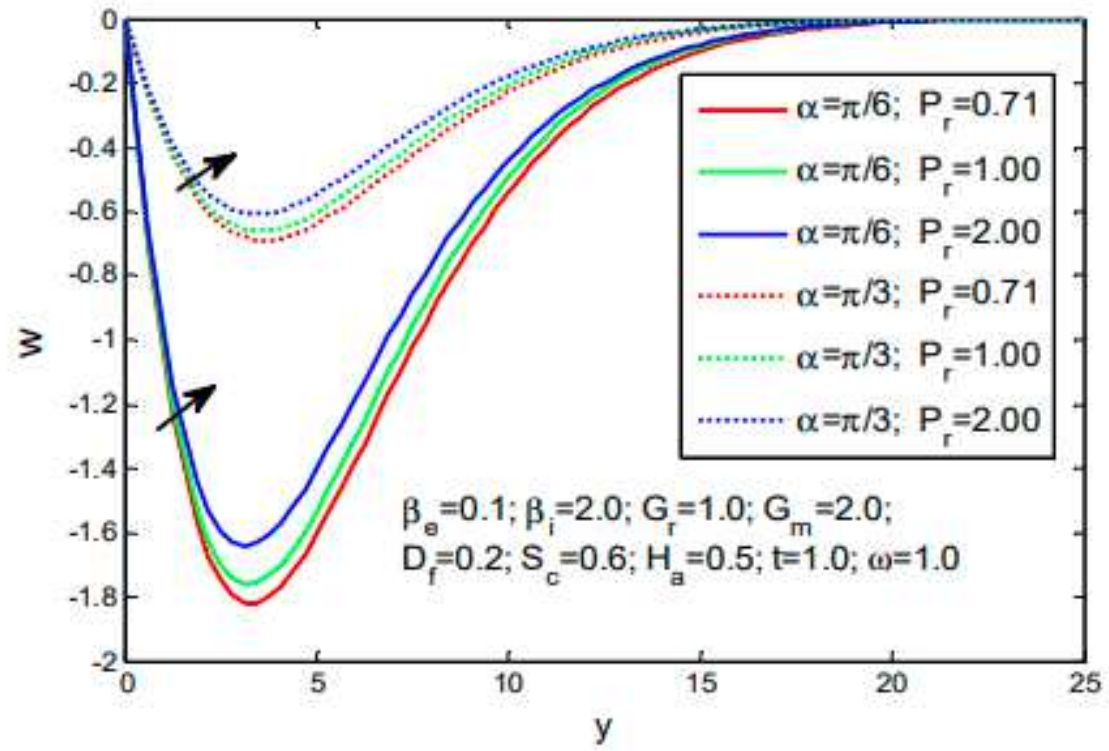

Figure 14

Please see the Manuscript PDF file for the complete figure caption 


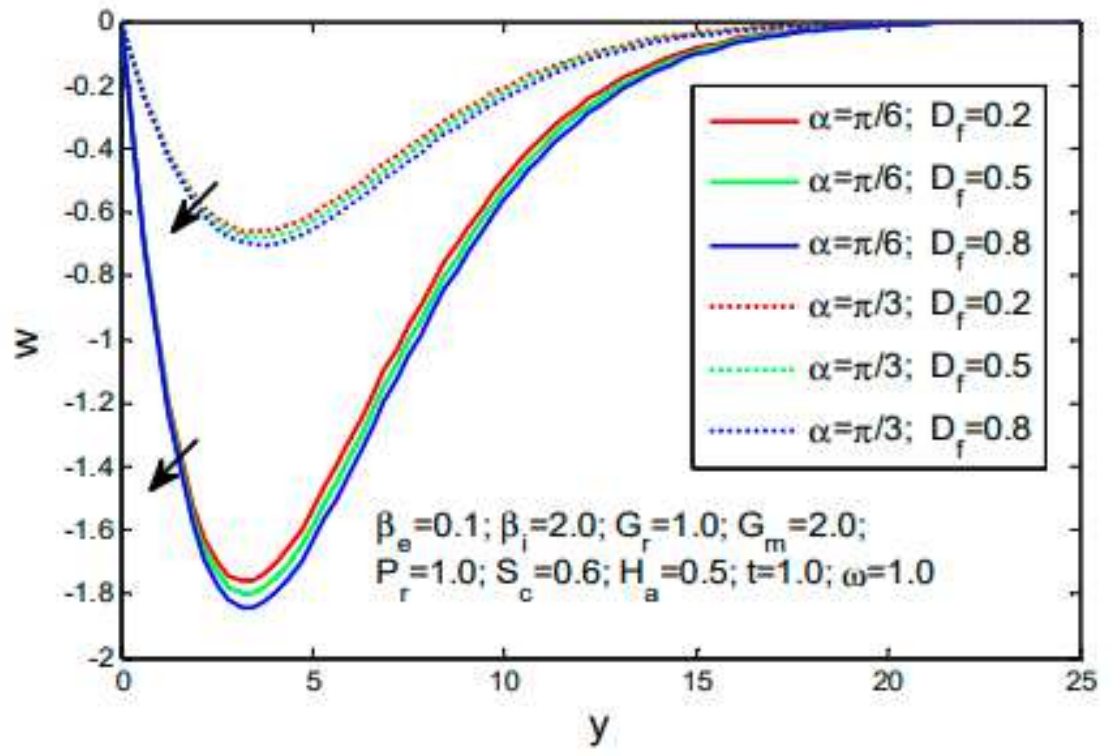

Figure 15

Please see the Manuscript PDF file for the complete figure caption

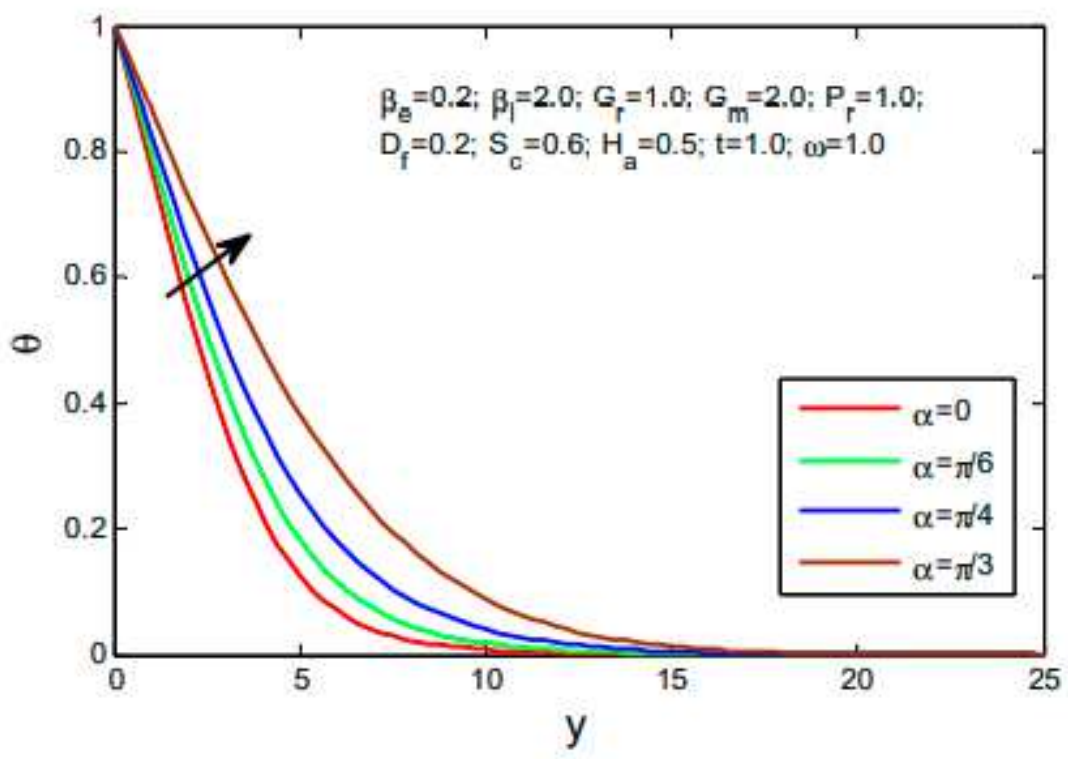

Figure 16

Please see the Manuscript PDF file for the complete figure caption 


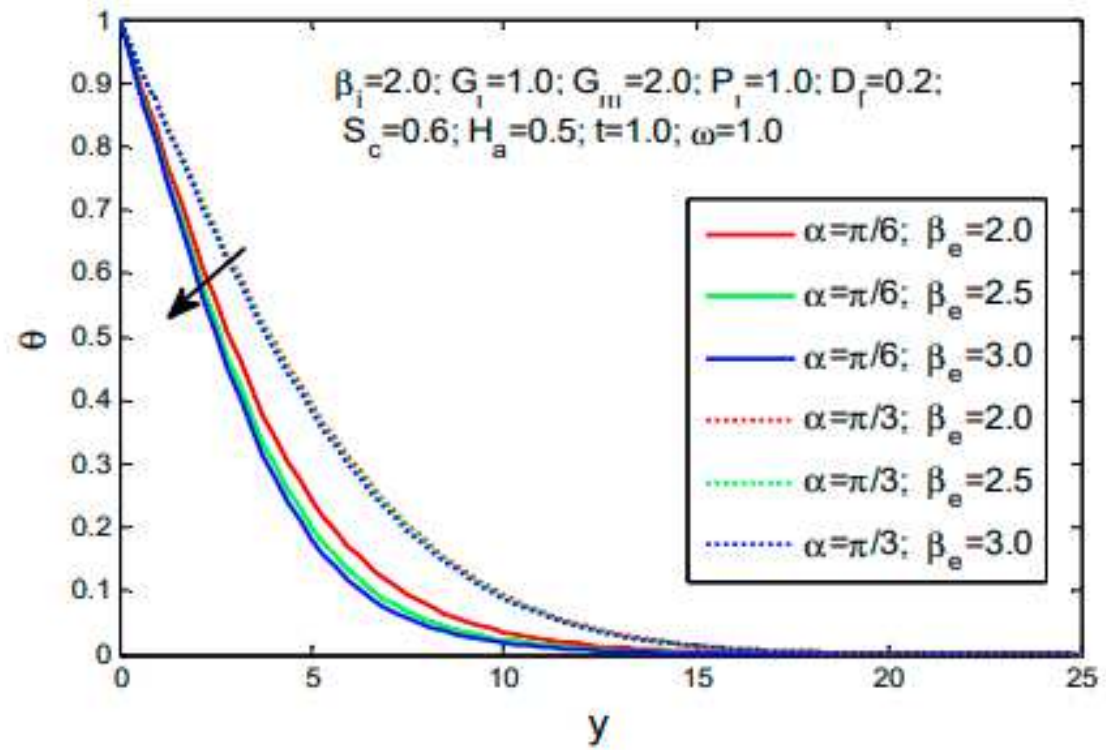

Figure 17

Please see the Manuscript PDF file for the complete figure caption

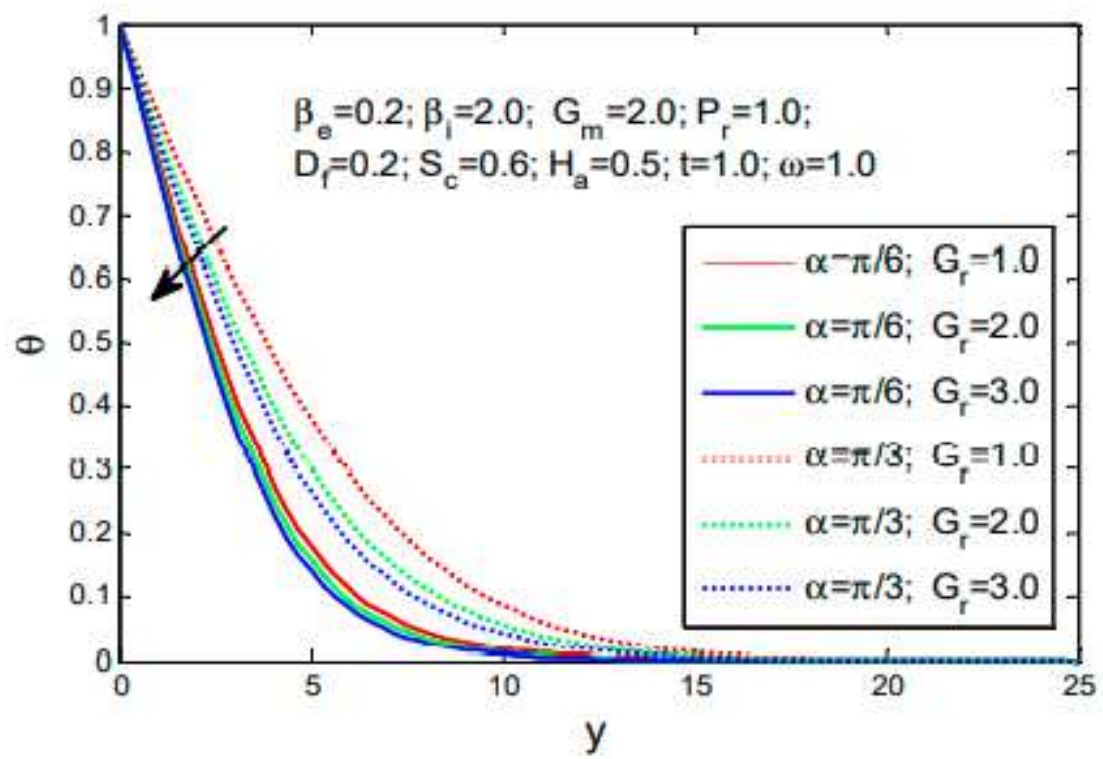

Figure 18

Please see the Manuscript PDF file for the complete figure caption 


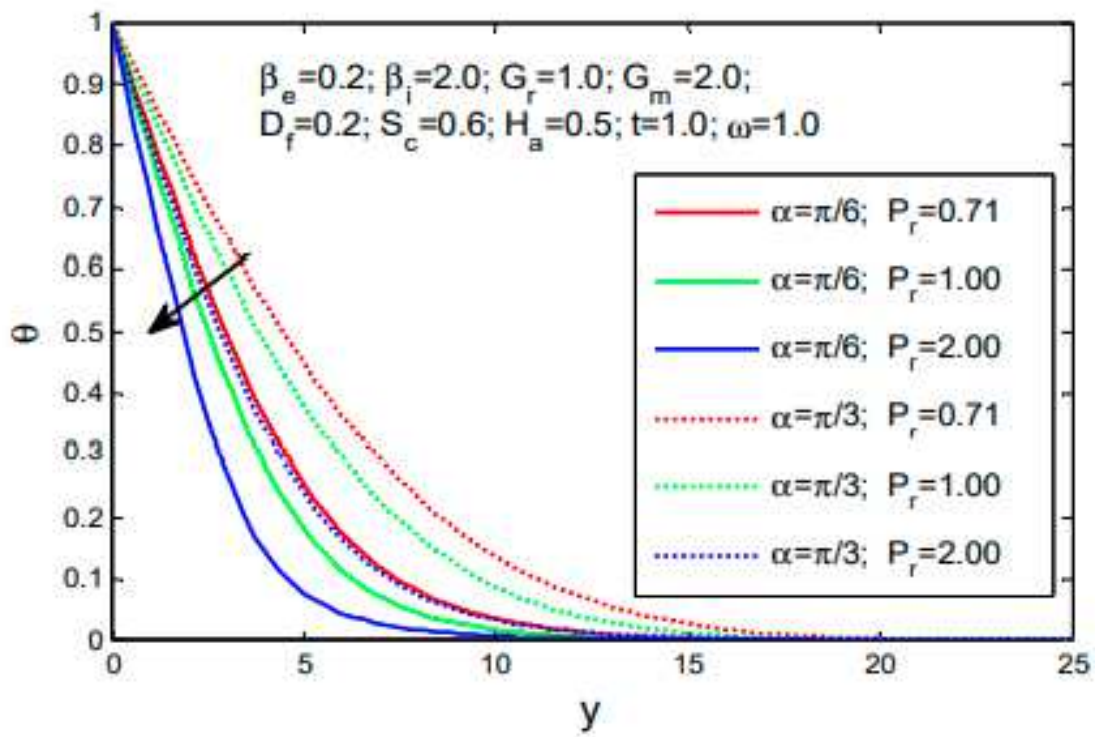

Figure 19

Please see the Manuscript PDF file for the complete figure caption

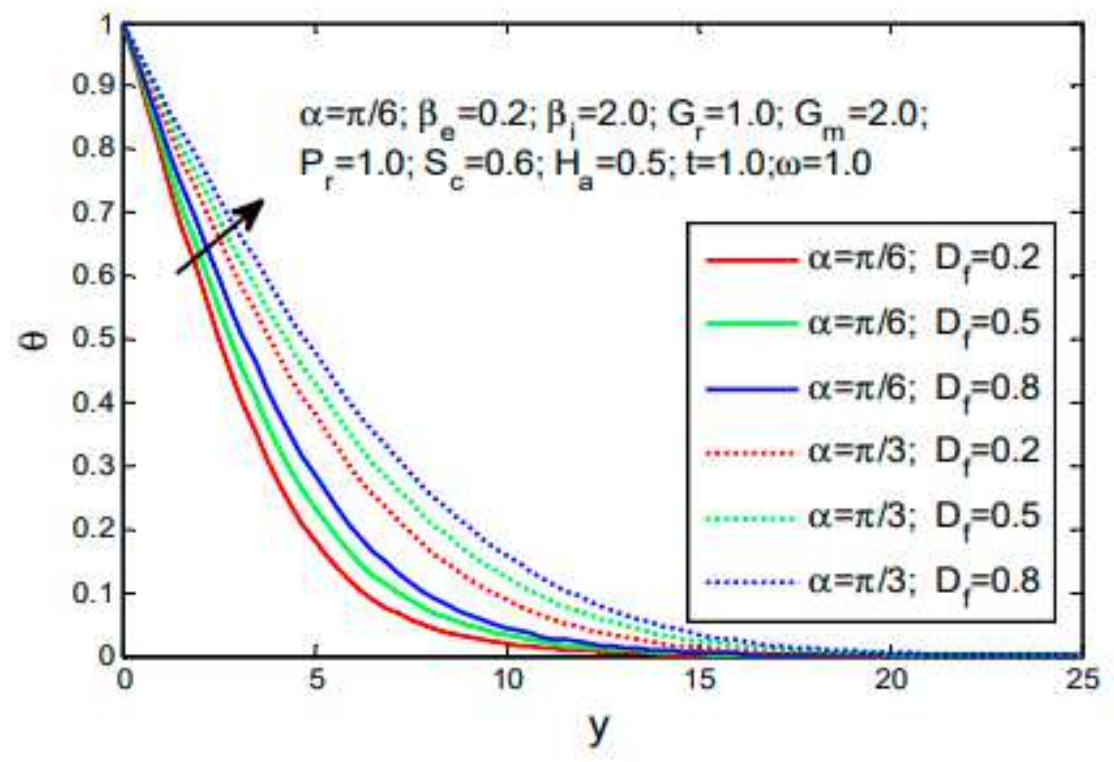

Figure 20

Please see the Manuscript PDF file for the complete figure caption 


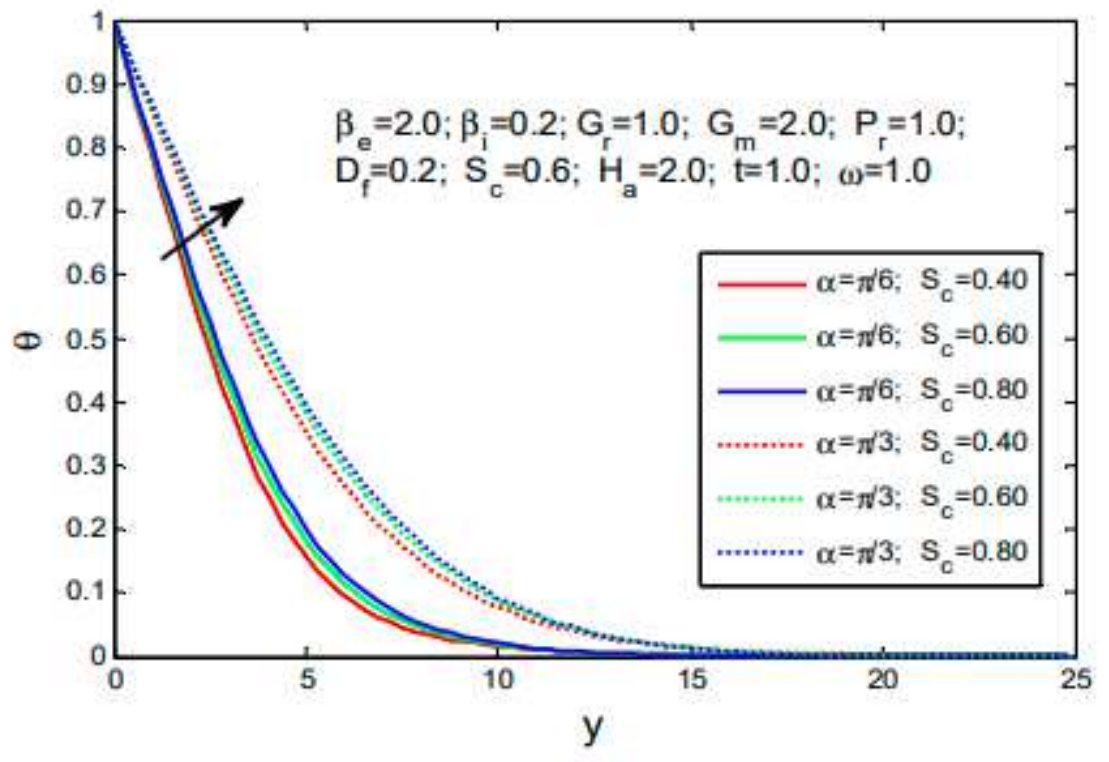

Figure 21

Please see the Manuscript PDF file for the complete figure caption

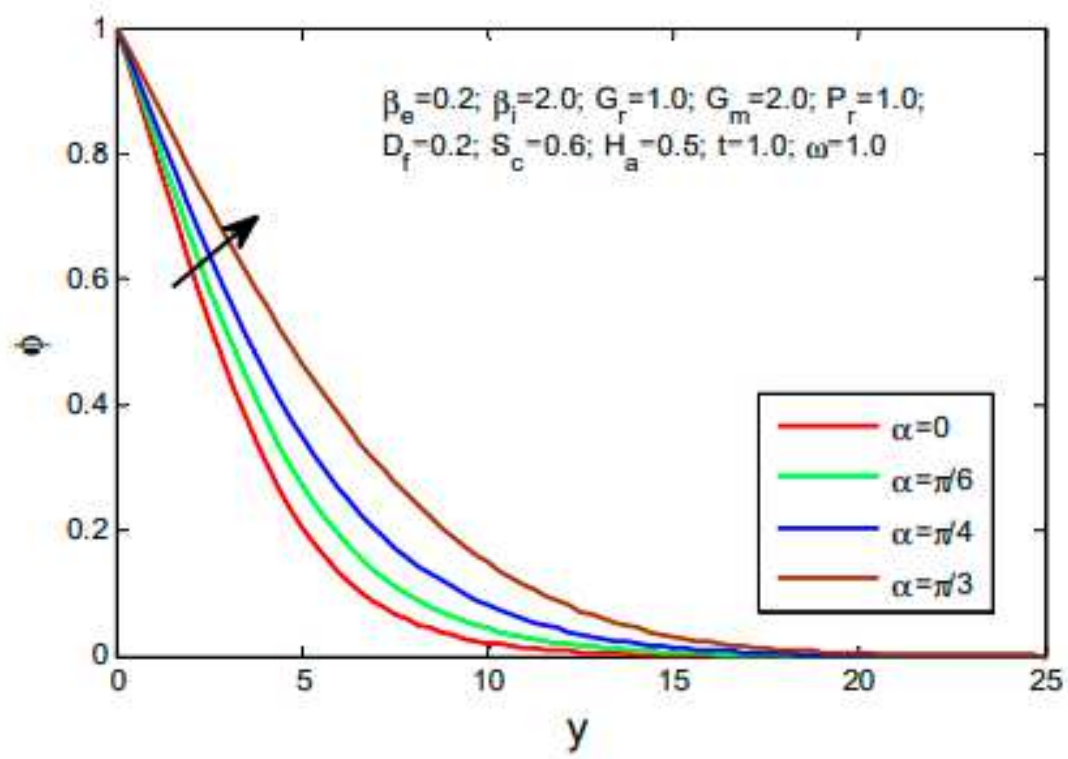

Figure 22

Please see the Manuscript PDF file for the complete figure caption 


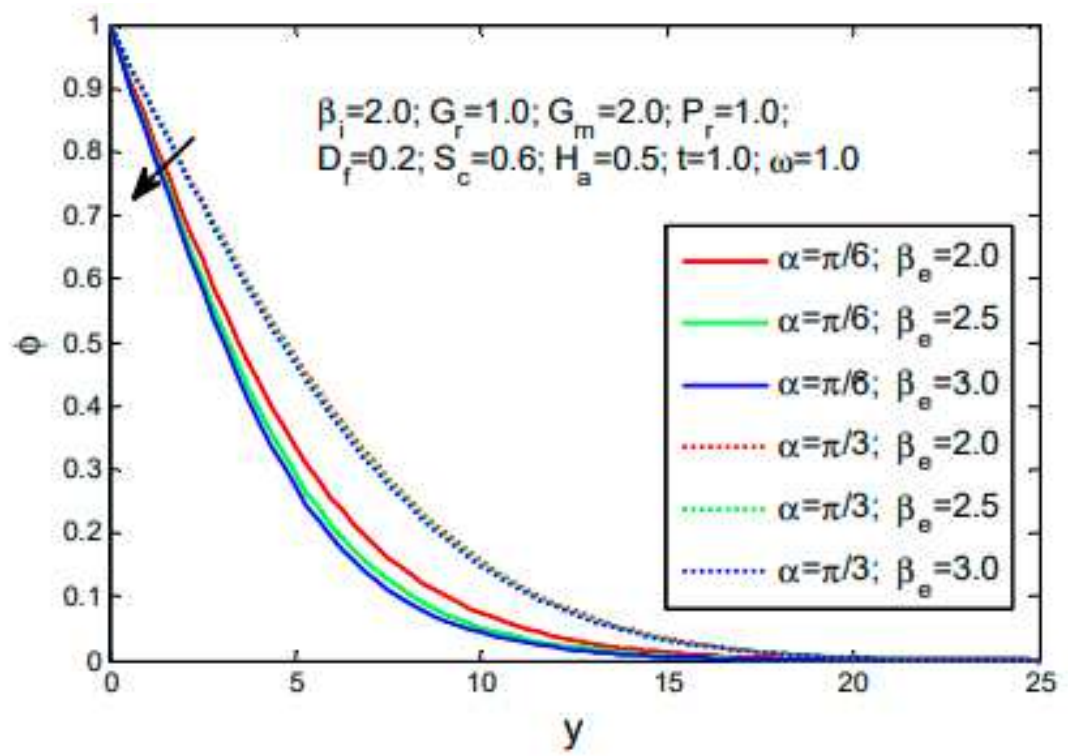

Figure 23

Please see the Manuscript PDF file for the complete figure caption

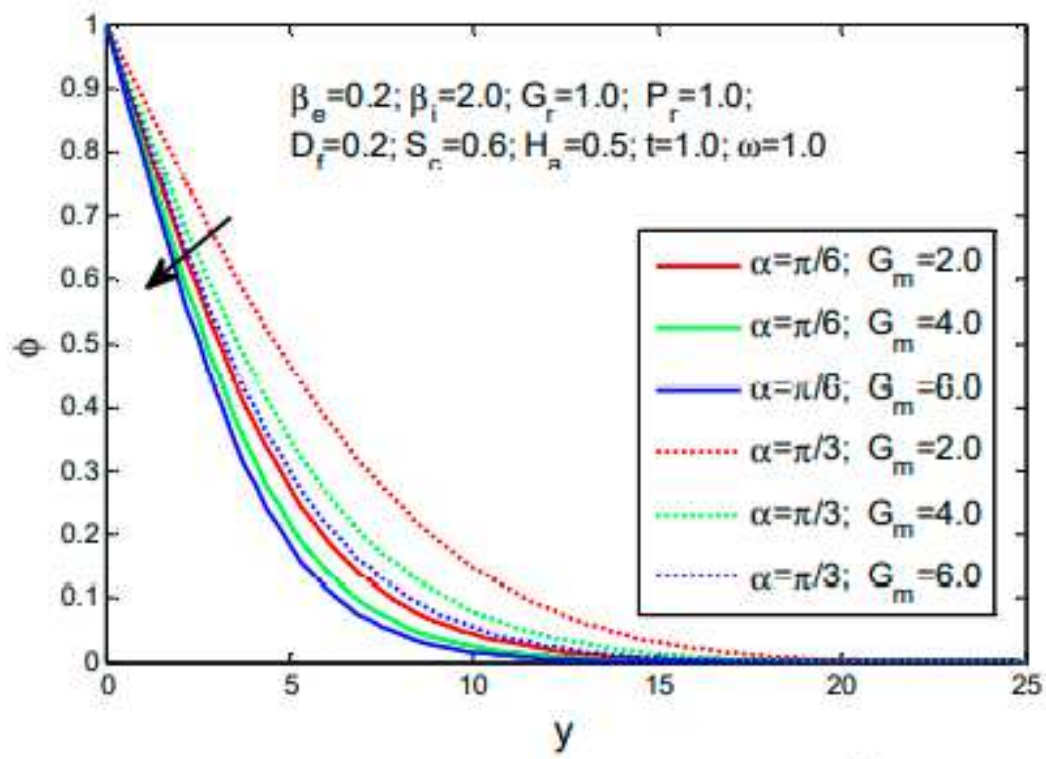

Figure 24

Please see the Manuscript PDF file for the complete figure caption 


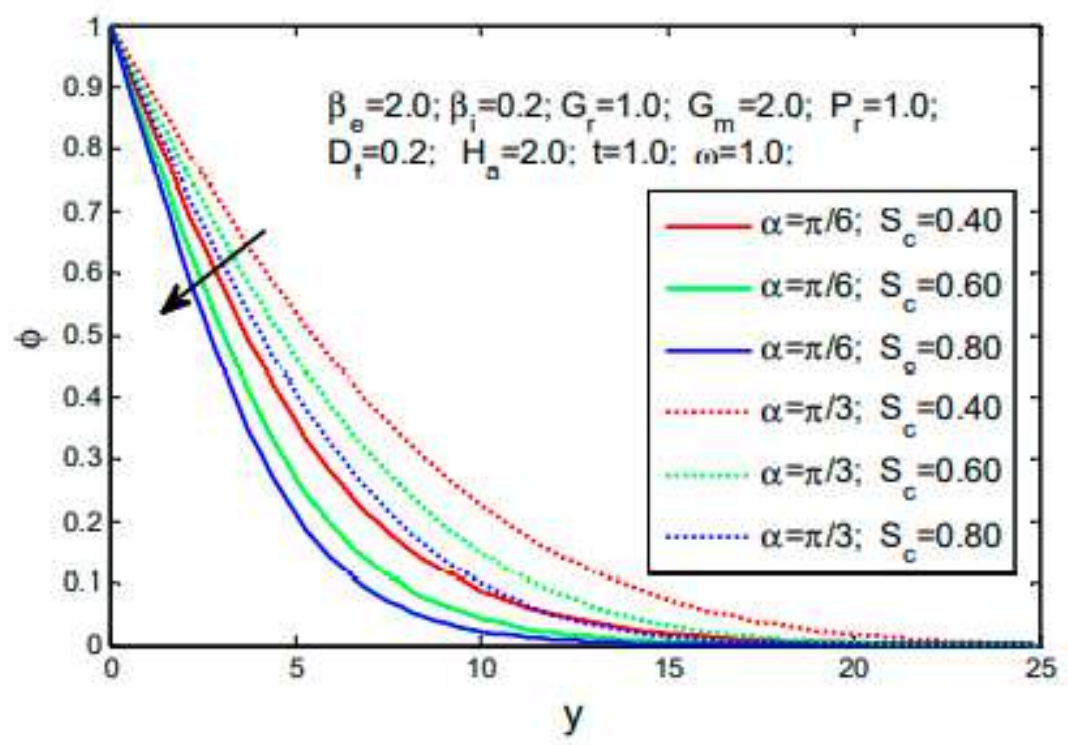

Figure 25

Please see the Manuscript PDF file for the complete figure caption
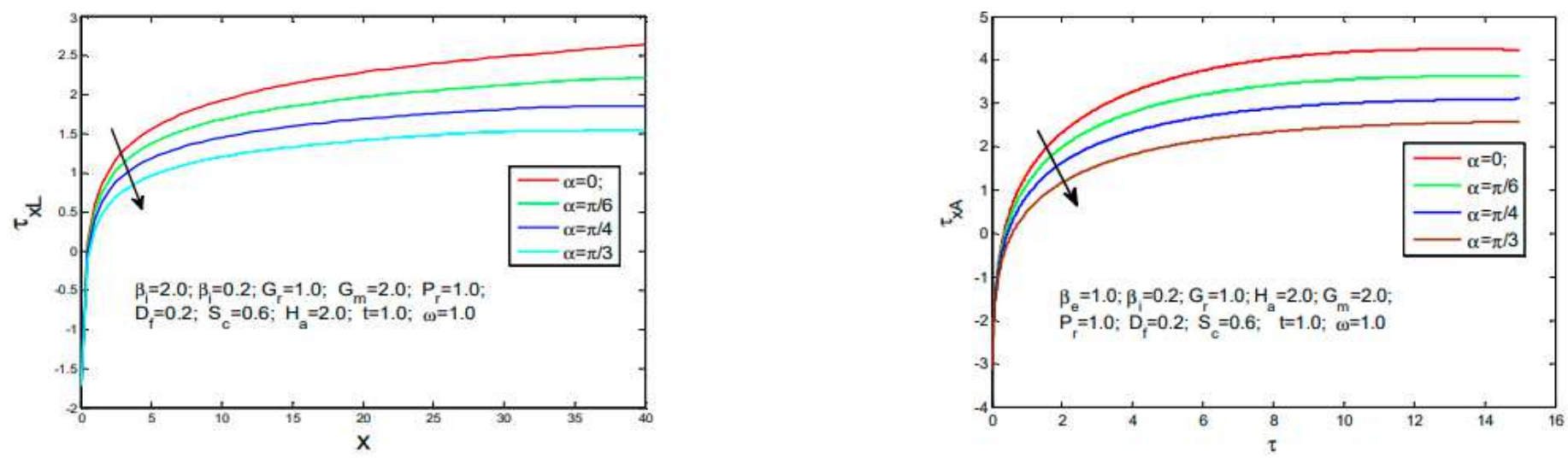

Figure 26

Please see the Manuscript PDF file for the complete figure caption
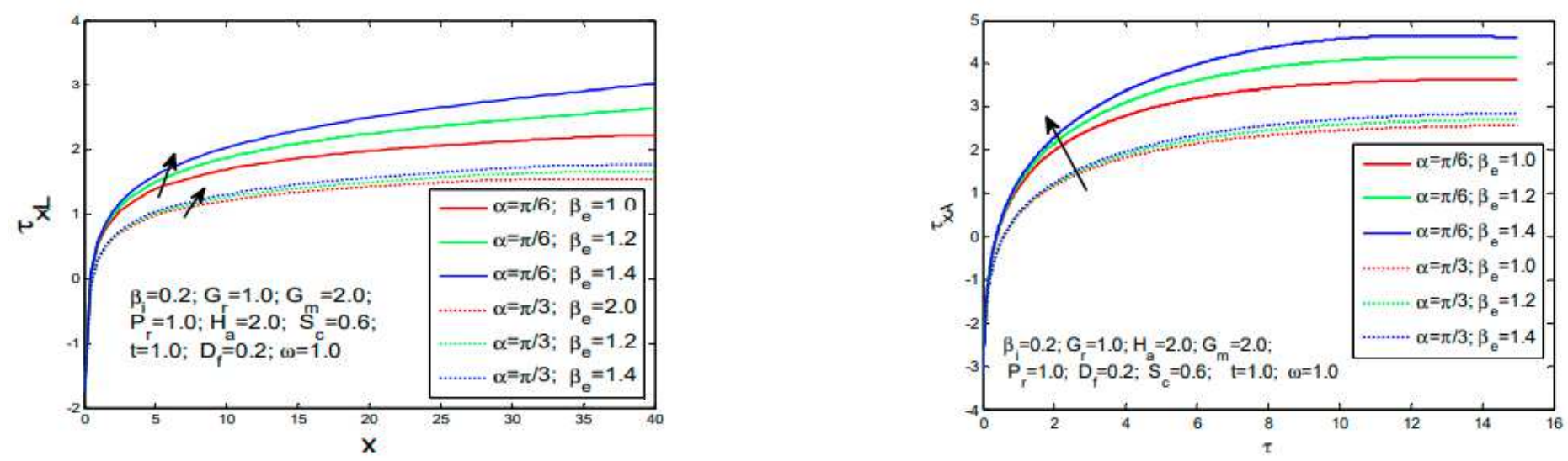
Figure 27

Please see the Manuscript PDF file for the complete figure caption
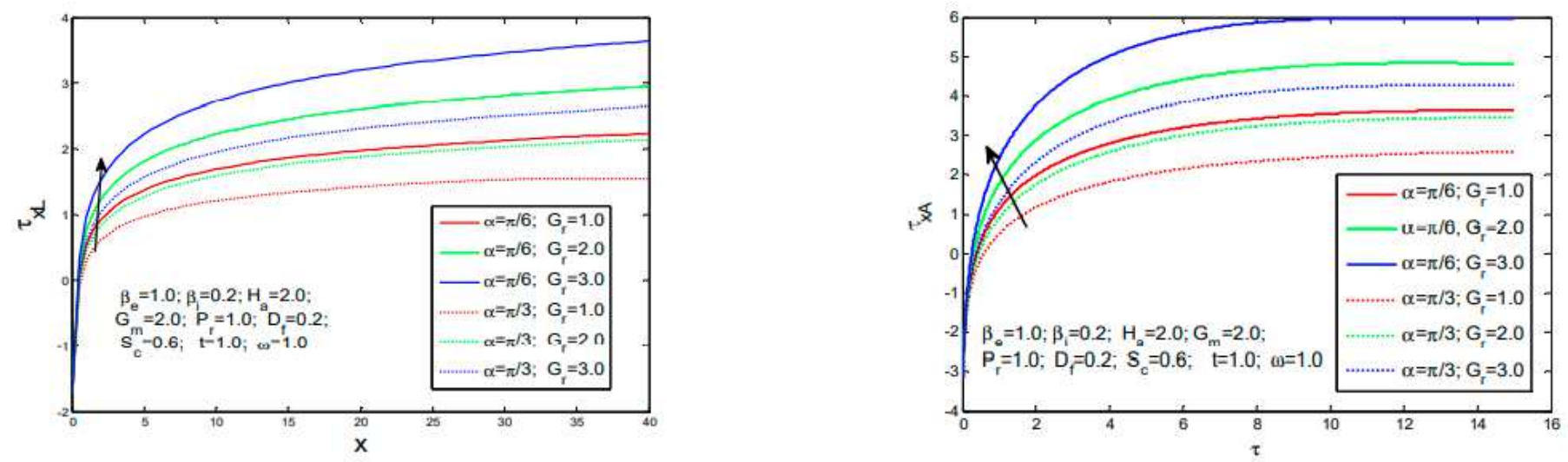

Figure 28

Please see the Manuscript PDF file for the complete figure caption
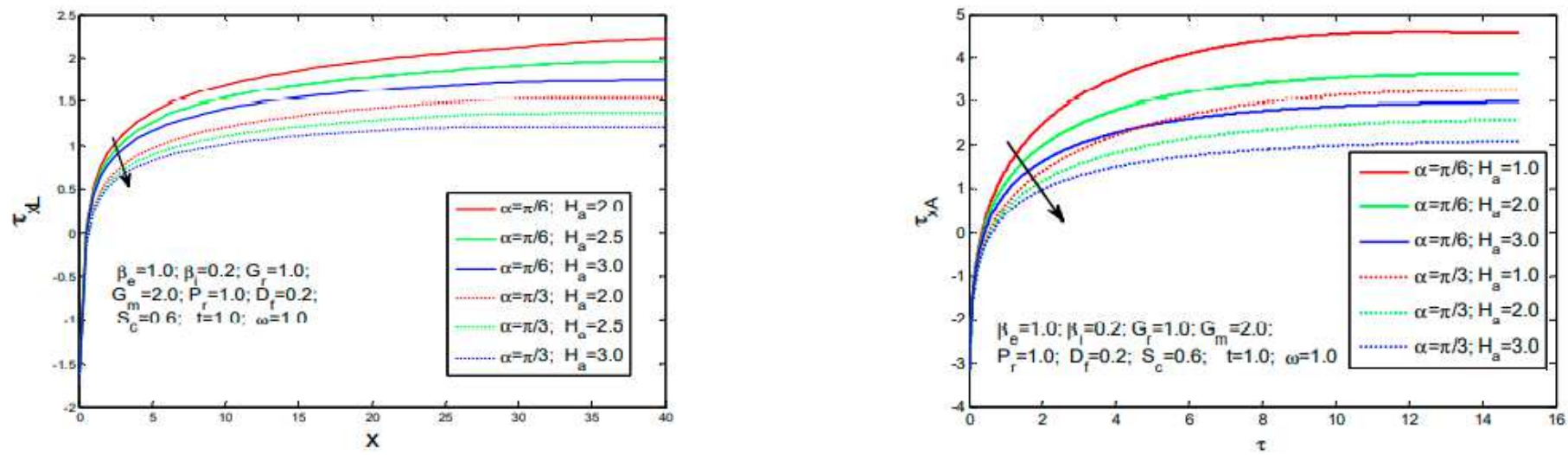

Figure 29

Please see the Manuscript PDF file for the complete figure caption
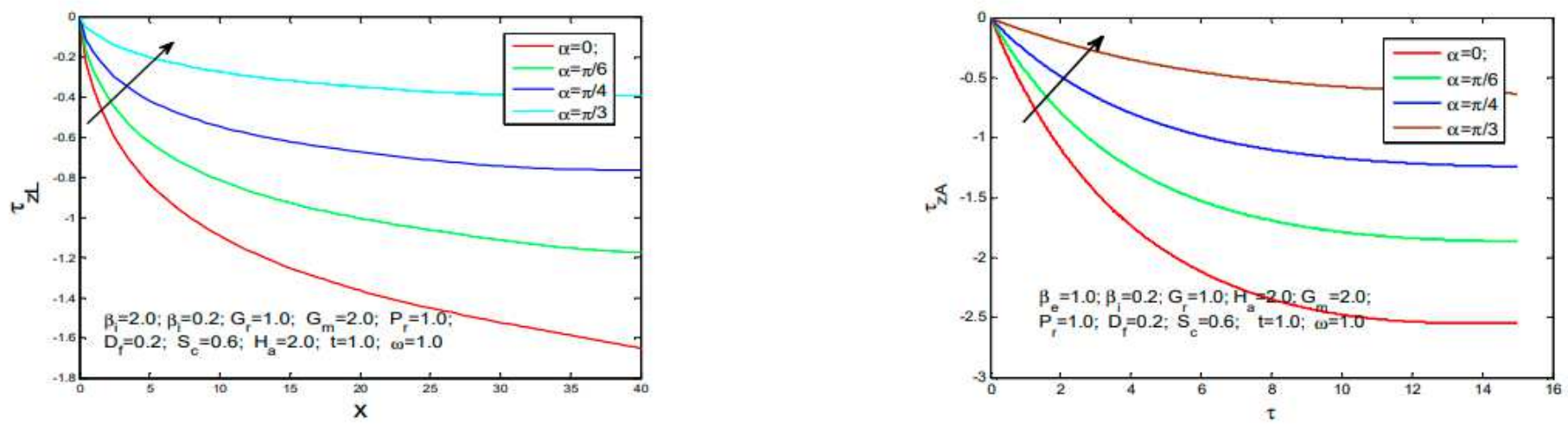

Figure 30 
Please see the Manuscript PDF file for the complete figure caption
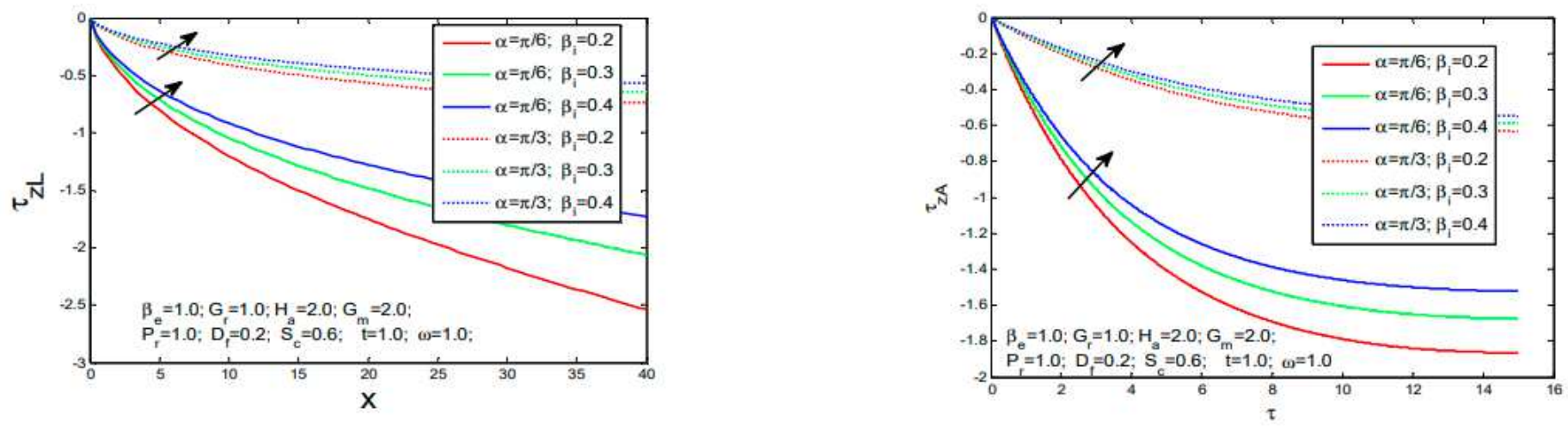

\section{Figure 31}

Please see the Manuscript PDF file for the complete figure caption
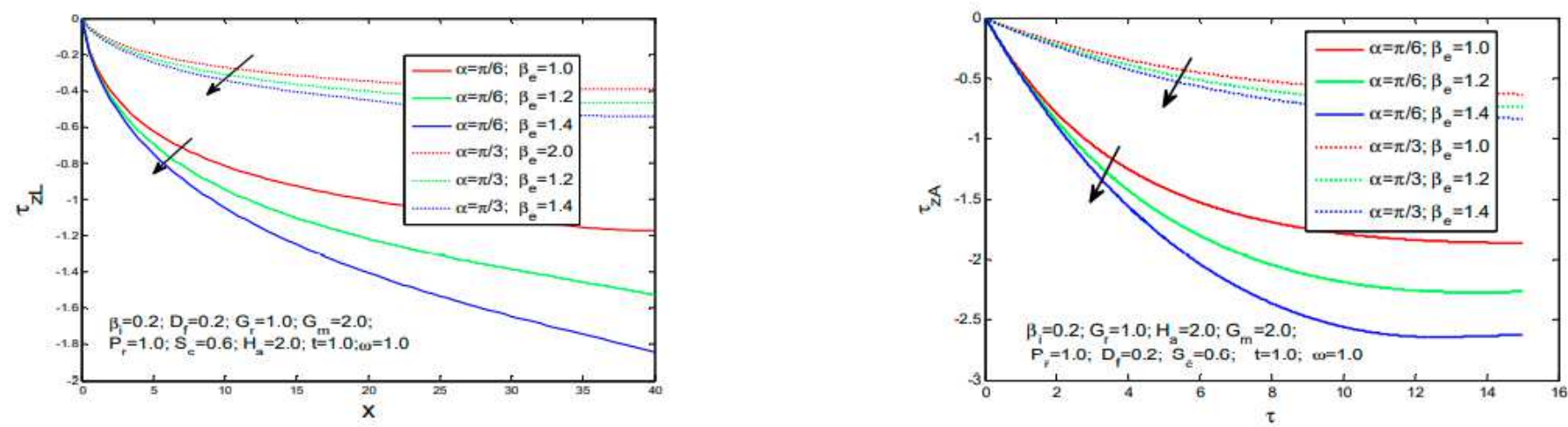

Figure 32

Please see the Manuscript PDF file for the complete figure caption
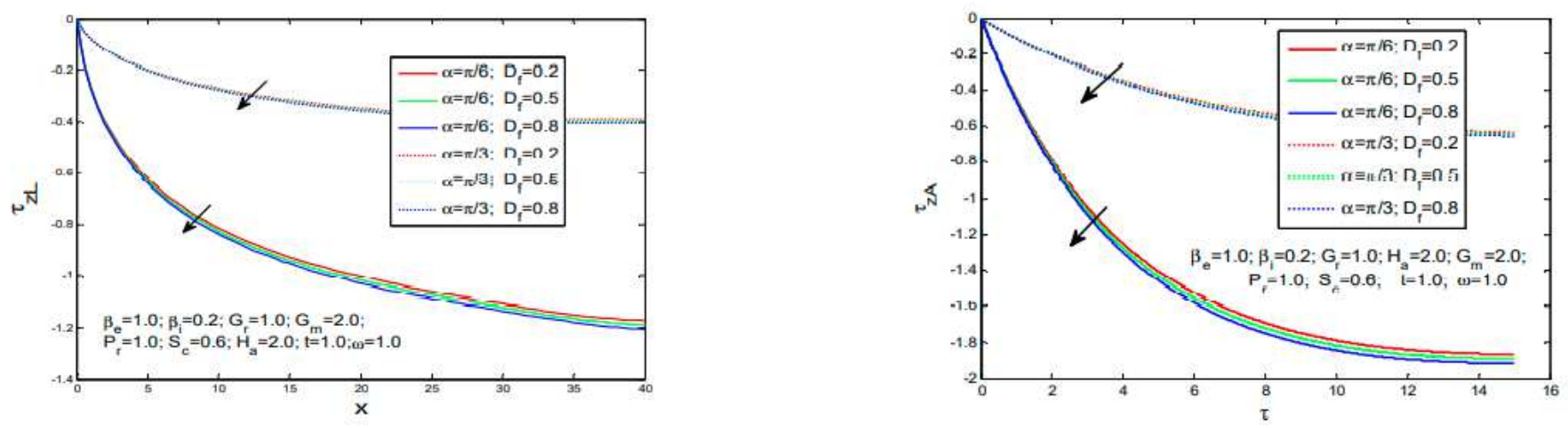

Figure 33

Please see the Manuscript PDF file for the complete figure caption 

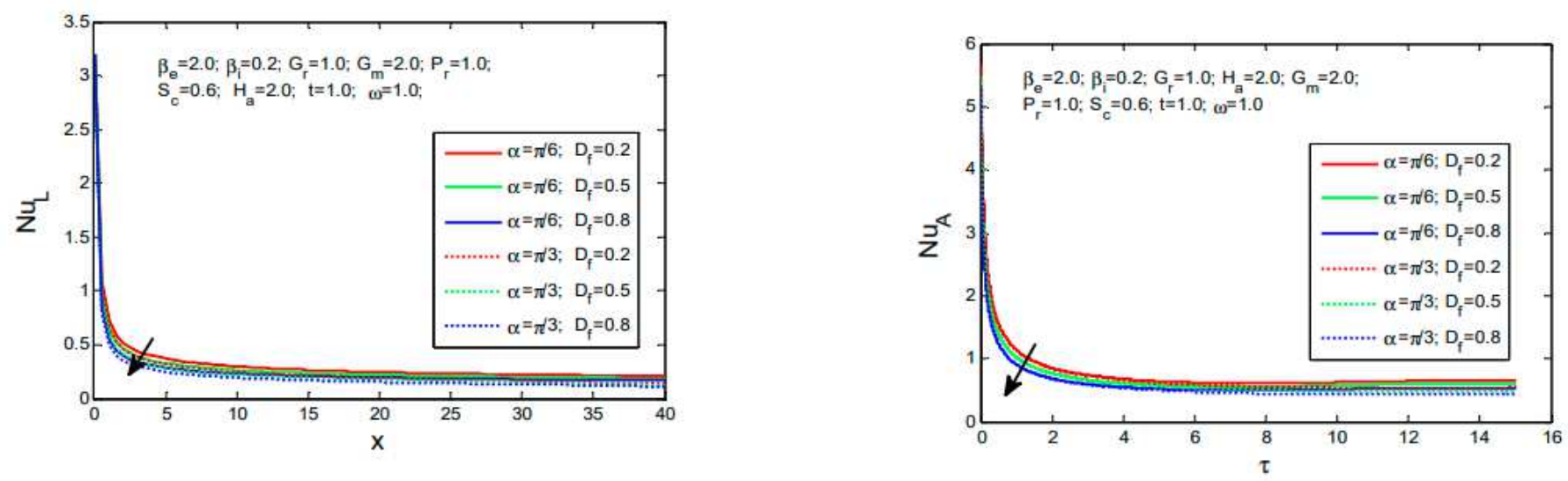

Figure 34

Please see the Manuscript PDF file for the complete figure caption
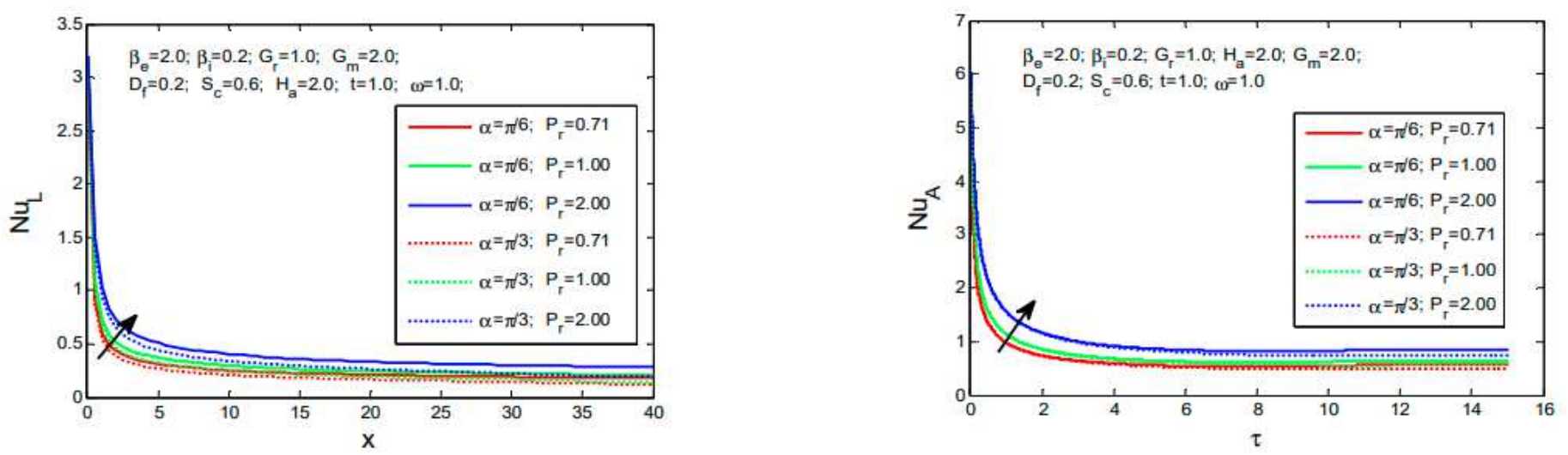

Figure 35

Please see the Manuscript PDF file for the complete figure caption
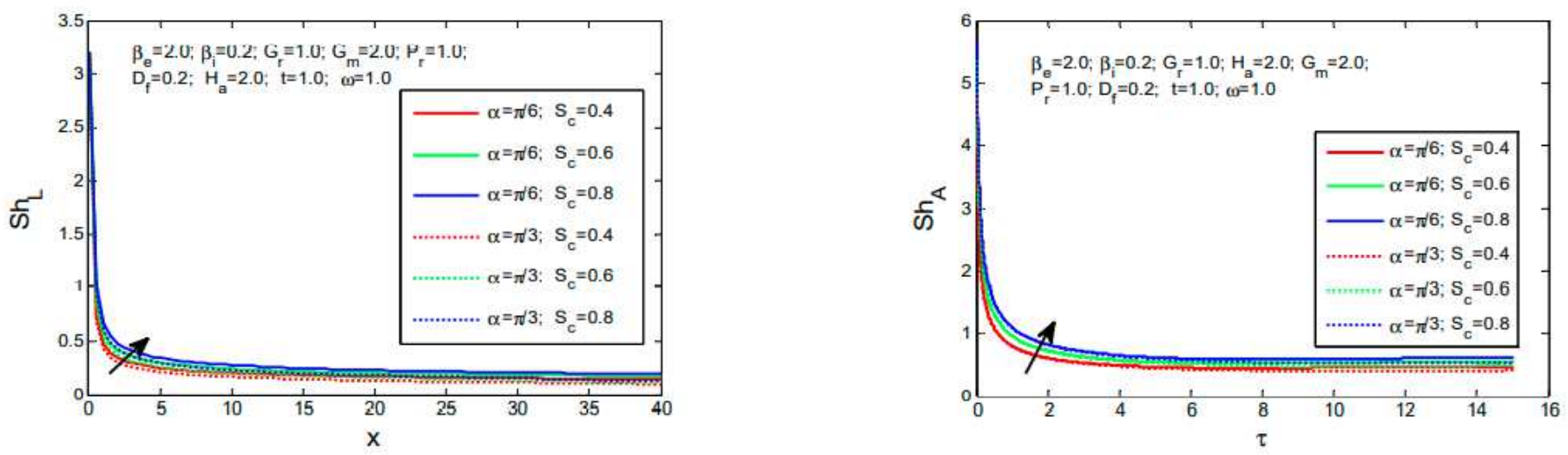

Figure 36

Please see the Manuscript PDF file for the complete figure caption 